

\title{
Polyhedral adjunction theory
}

\author{
Sandra Di Rocco, Christian Haase, Benjamin Nill and Andreas Paffenholz
}

\begin{abstract}
In this paper we offer a combinatorial view on the adjunction theory of toric varieties. Inspired by classical adjunction theory of polarized algebraic varieties we explore two convex-geometric notions: the $\mathbb{Q}$-codegree and the nef value of a rational polytope $P$. We prove a structure theorem for lattice polytopes $P$ with large $\mathbb{Q}$-codegree. For this, we define the adjoint polytope $P^{(s)}$ as the set of those points in $P$ whose lattice distance to every facet of $P$ is at least $s$. It follows from our main result that if $P^{(s)}$ is empty for some $s<2 /(\operatorname{dim} P+2)$, then the lattice polytope $P$ has lattice width one. This has consequences in Ehrhart theory and on polarized toric varieties with dual defect. Moreover, we illustrate how classification results in adjunction theory can be translated into new classification results for lattice polytopes.
\end{abstract}

\section{Introduction}

Let $P \subseteq \mathbb{R}^{n}$ be a rational polytope of dimension $n$. Any such polytope $P$ can be described in a unique minimal way as

$$
P=\left\{x \in \mathbb{R}^{n}:\left\langle a_{i}, x\right\rangle \geqslant b_{i}, i=1, \ldots, m\right\},
$$

where the $a_{i}$ are primitive rows of an $m \times n$ integer matrix $A$ and $b \in \mathbb{Q}^{m}$.

For any $s \geqslant 0$ we define the adjoint polytope $P^{(s)}$ as

$$
P^{(s)}:=\left\{x \in \mathbb{R}^{n}: A x \geqslant b+s \mathbb{1}\right\},
$$

where $\mathbb{1}=(1, \ldots, 1)^{\mathrm{T}}$.

We call the study of such polytopes $P^{(s)}$ polyhedral adjunction theory.

Adjunction theory is an area of algebraic geometry which has played a fundamental role in the classification of projective algebraic varieties [Batyrev and Tschinkel 1998; Beltrametti and Di Termini 2003; Beltrametti et al. 1992; Beltrametti and

Di Rocco has been partially supported by VR-grants NT:2006-3539 and NT:2010-5563. Haase and Nill were supported by Emmy Noether fellowship HA 4383/1 and Heisenberg fellowship HA 4383/4 of the German Research Society (DFG). Nill is supported by the US National Science Foundation (DMS 1203162). Paffenholz is supported by the Priority Program 1489 of the German Research Foundation.

MSC2010: primary 14C20; secondary 14M25, 52B20.

Keywords: convex polytopes, toric varieties, adjunction theory. 

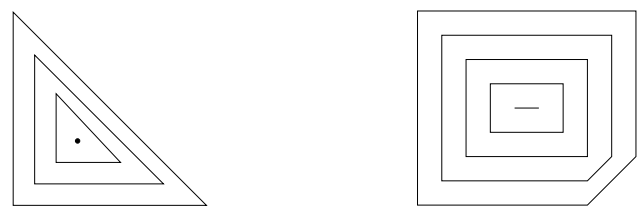

Figure 1. Two examples of polyhedral adjunction. See page 2426 for discussion.

Sommese 1994; 1995; Fania and Sommese 1989; Fujita 1987; 1992; 1996; 1997; Nakamura 1997; Sommese 1986]. The correspondence between polarized toric varieties and lattice polytopes provides a natural ground for an adjunction theory of lattice polytopes, as suggested in [Dickenstein et al. 2009].

The main purpose of this article is to convince the reader that polyhedral adjunction theory is an exciting area of research with many open questions connecting toric geometry, polyhedral combinatorics and geometry of numbers.

By the toric dictionary between convex geometry of polytopes and geometry of projective toric varieties, a lattice polytope $P$ defines a toric variety $X_{P}$ polarized by an ample line bundle $L_{P}$. The pair $\left(X_{P}, L_{P}\right)$ is often referred to as a polarized toric variety. Sometimes the pair $(X, L)$ is replaced by the equivariant embedding $X \hookrightarrow \mathbb{P}^{N}$ defined by a suitable multiple of the line bundle $L$. Adjunction theory provides tools to characterize and classify the pairs $(X, L)$ by looking at the behavior of the adjoint systems $\left|u K_{X}+v L\right|$, for integers $u, v$, where $K_{X}$ is the canonical divisor in $X$. We refer to Section 4 for details. If $P$ is the polytope defined by the line bundle $L$ on $X$, then $(v P)^{(u)}$ is the polytope defined by the line bundle $u K_{X}+v L$.

In adjunction theory the nef value $\tau(L)$ and the unnormalized spectral value $\mu(L)$ (sometimes called the canonical threshold) measure the positivity of the adjoint systems. In Section 4 an account of these notions is given. An "integral" version of the unnormalized spectral value for lattice polytopes has been present in the literature for quite some time (even though it was never defined this way) under the name codegree, denoted by $\operatorname{cd}(P)$ - see Definition 1.7. This notion appeared in connection with Ehrhart theory and was studied by Batyrev and Nill [2007].

A "rational" version, again for lattice polytopes, has recently been introduced in [Dickenstein et al. 2009]. Let $c$ be the maximal rational number for which $P^{(c)}$ is nonempty. Its reciprocal $\mu(P):=1 / c$ equals precisely the unnormalized spectral value $\mu\left(L_{P}\right)$. It is called the $\mathbb{Q}$-codegree of $P$ (Definition 1.5).

A long-standing conjecture in algebraic geometry states that general polarized varieties should have unnormalized spectral values that are bounded above by approximately half their dimension. In particular, as discussed more fully in Remark 4.10, we have the following conjecture: 
Conjecture 1 [Beltrametti and Sommese 1994]. If an $n$-dimensional polarized variety $X$ is smooth, then $\mu(L)>(n+1) / 2$ implies that $X$ is a fibration.

Let us consider lattice polytopes again. A Cayley sum of $t+1$ polytopes is a polytope (denoted by $P_{0} * \cdots * P_{t}$ ) built by assembling the polytopes $P_{i}$ along the vertices of a $t$-dimensional simplex - see Definition 3.1. For $t=0$, the condition of being a Cayley sum is vacuous. So when we say that $P$ has a Cayley structure we mean a nontrivial one with $t>0$. For example, for $t=1$, the condition is known in the literature as $P$ having lattice width one. From an (apparently) unrelated perspective Batyrev and Nill conjectured that there is a function $f(n)$ such that, if $\operatorname{cd}(P) \geqslant f(n)$, the polytope has a nontrivial Cayley structure. This can be sharpened:

Conjecture 2 [Dickenstein and Nill 2010]. If an $n$-dimensional lattice polytope $P$ satisfies $\operatorname{cd}(P)>(n+2) / 2$, then $P$ decomposes as a Cayley sum of lattice polytopes of dimension at most $2(n+1-\operatorname{cd}(P))$.

The polarized toric variety associated to a Cayley polytope is birationally fibered in projective spaces, as explained on page 2441. It follows that Conjecture 2 could be considered an "integral-toric" version of Conjecture 1 extended to singular varieties. It also suggests that geometrically it would make sense to replace $\operatorname{cd}(P)$ by $\mu(P)$ and use the bound $(n+1) / 2$ from Conjecture 1 . This leads to a reformulation (we note that $\mu(P) \leqslant \operatorname{cd}(P))$ :

Conjecture 3. If an $n$-dimensional lattice polytope $P$ satisfies $\mu(P)>(n+1) / 2$, then $P$ decomposes as a Cayley sum of lattice polytopes of dimension at most $\lfloor 2(n+1-\mu(P))\rfloor$.

The main result of this paper is Theorem 3.4. It implies a slightly weaker version of Conjecture 3, with $\mu(P)>(n+1) / 2$ replaced by $\mu(P) \geqslant(n+2) / 2-$ see Corollary 3.7.

Despite much work both Conjectures 1 and 2 are still open in their original generality. It is known that $f(n)$ can be chosen quadratic in $n$ [Haase et al. 2009] and that Conjecture 2 is true for smooth polytopes [Dickenstein et al. 2009; Dickenstein and Nill 2010]. The results in [Dickenstein et al. 2009; Dickenstein and Nill 2010] also imply that for toric polarized manifolds Conjecture 1 holds for $\mu(L)>(n+2) / 2$.

Besides the underlying geometric intuition and motivation, polyhedral adjunction theory and the results of this paper have connections with other areas.

Geometry of numbers. It follows from the definition of the $\mathbb{Q}$-codegree that $\mu(P)>1$ implies that $P$ is lattice-free, that is, it has no interior lattice points. Lattice-free polytopes are of importance in geometry of numbers and integer linear optimization see [Averkov et al. 2011; Nill and Ziegler 2011] for recent results. Lattice-free simplices turn up naturally in singularity theory [Morrison and Stevens 1984]. Most 
prominently, the famous flatness theorem states that $n$-dimensional lattice-free convex bodies have bounded lattice width (we refer to [Barvinok 2002] for details). Cayley polytopes provide the most special class of lattice-free polytopes: they have lattice width one, that is, the vertices of the polytope lie on two parallel affine hyperplanes that do not have any lattice points lying strictly between them. Our main result, Theorem 3.4, shows that lattice polytopes with sufficiently large $\mathbb{Q}$-codegree have to be Cayley polytopes. This hints at a close and not yet completely understood relation between the $\mathbb{Q}$-codegree and the lattice width of a lattice polytope.

Let us remark that for $n \geqslant 3$ Corollary 3.7 only provides a sufficient criterion for $P$ to be a Cayley polytope. For instance, $P=[0,1]^{n}$ has lattice width one, but $\mu(P)=2<(n+2) / 2$. Still, for even $n$ the choice of $(n+2) / 2$ is tight. Let $P=2 \Delta_{n}$, where $\Delta_{n}:=\operatorname{conv}\left(0, e_{1}, \ldots, e_{n}\right)$ is the unimodular $n$-simplex. Here, $P$ does not have lattice width one, since every edge contains a lattice point in the middle. On the other hand, we have $\mu(P)=(n+1) / 2$. Since for $n$ even we have $\operatorname{cd}(P)=(n+2) / 2$, this example also shows that the bound $(n+2) / 2$ in Conjecture 2 is sharp.

Projective duality. There is evidence that the unnormalized spectral value is connected to the behavior of the associated projective variety under projective duality. An algebraic variety is said to be dual defective if its dual variety has codimension strictly larger than 1 . The study of dual defective projective varieties is a classical area of algebraic geometry (starting from Bertini) and a growing subject in combinatorics and elimination theory, as it is related to discriminants [Gelfand et al. 1994]. It is known that nonsingular dual-defective polarized varieties necessarily satisfy $\mu>(n+2) / 2$ [Beltrametti et al. 1992]. On the other hand, in [Dickenstein and Nill 2010; Di Rocco 2006] it was shown that a polarized nonsingular toric variety corresponding to a lattice polytope $P$ as above is dual defective if and only if $\mu>(n+2) / 2$. It was conjectured in [Dickenstein and Nill 2010] that also in the singular toric case $\mu>(n+2) / 2$ would imply $\left(X_{P}, L_{P}\right)$ to be dual defective. Theorem 3.4 gives significant evidence in favor of this conjecture, as it was shown in [Curran and Cattani 2007; Esterov 2010] that the lattice points in such a dual defective lattice polytope lie on two parallel hyperplanes. Moreover, using our main result we verify a weaker version of this conjecture (Proposition 4.11).

Classification of polytopes and adjunction theory beyond $\mathbb{Q}$-Gorenstein varieties. We believe that polyhedral adjunction theory can help to develop useful intuition for problems in (not necessarily toric) classical adjunction theory, when no algebrogeometric tools or results exist so far. For instance, defining $\mu$ makes sense in the polyhedral setting even if the canonical divisor of the toric variety is not $\mathbb{Q}$-Cartier.

How to read this paper. Sections 1-3, as well as the Appendix, are kept purely combinatorial, no prior knowledge of algebraic or toric geometry is assumed. The 
algebro-geometrically inclined reader may jump directly to Section 4 . We refer the reader who is unfamiliar with polytopes to [Ziegler 1995].

In Section 1 we introduce the two main players: the $\mathbb{Q}$-codegree and the nef value of a rational polytope. Section 2 proves useful results about how these invariants behave under (natural) projections. These results should be viewed as a toolbox for future applications. Section 3 contains the main theorem and its proof. The algebro-geometric background and implications are explained in Section 4. In the Appendix we include a combinatorial translation of some well-known algebrogeometric classification results by Fujita which we think may be of interest to combinatorialists.

\section{The $\mathbb{Q}$-codegree, the codegree, and the nef value}

Throughout let $P \subseteq \mathbb{R}^{n}$ be an $n$-dimensional rational polytope.

Preliminaries. Let us recall that $P$ is a rational polytope if the vertices of $P$ lie in $\mathbb{Q}^{n}$. Moreover, $P$ is a lattice polytope if its vertices lie in $\mathbb{Z}^{n}$. We consider lattice polytopes up to lattice-preserving affine transformations. Let us denote by $\langle\cdot, \cdot\rangle$ the pairing between $\mathbb{Z}^{n}$ and its dual lattice $\left(\mathbb{Z}^{n}\right)^{*}$.

There exists a natural lattice distance function $d_{P}$ on $\mathbb{R}^{n}$ such that for $x \in \mathbb{R}^{n}$ the following holds: $x \in P$ (respectively, $x \in \operatorname{int}(P)$ ) if and only if $d_{P}(x) \geqslant 0$ (respectively, $d_{P}(x)>0$ ).

Definition 1.1. Let $P$ be given by the inequalities

$$
\left\langle a_{i}, \cdot\right\rangle \geqslant b_{i} \quad \text { for } i=1, \ldots, m,
$$

where $b_{i} \in \mathbb{Q}$ and the $a_{i} \in\left(\mathbb{Z}^{n}\right)^{*}$ are primitive (i.e., they are not the multiple of another lattice vector). We consider the $a_{i}$ as the rows of an $m \times n$ integer matrix $A$. Further, we assume all inequalities to define facets $F_{i}$ of $P$. Then for $x \in \mathbb{R}^{n}$ we define the lattice distance from $F_{i}$ by

$$
d_{F_{i}}(x):=\left\langle a_{i}, x\right\rangle-b_{i}
$$

and the lattice distance with respect to $\partial P$ by

$$
d_{P}(x):=\min _{i=1, \ldots, m} d_{F_{i}}(x) .
$$

For $s>0$ we define the adjoint polytope as

$$
P^{(s)}:=\left\{x \in \mathbb{R}^{n}: d_{P}(x) \geqslant s\right\} .
$$

Remark 1.2. We remark that it is important to assume that all $F_{i}$ are facets, as the following two-dimensional example shows. Let $a_{1}:=(-1,1), a_{2}:=(1,2)$, $a_{3}:=(0,-1), a_{4}:=(0,1)$. We set $b_{1}:=0, b_{2}:=0, b_{3}:=-1, b_{4}:=0$. This defines 


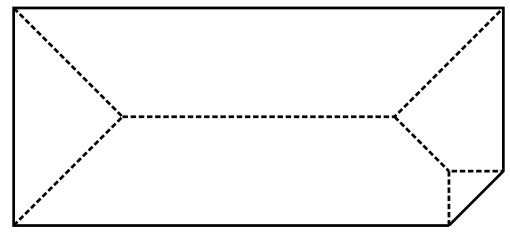

Figure 2. The skeleton of vertices of the adjoint polytopes.

the lattice triangle $P:=\operatorname{conv}((0,0),(1,1),(-2,1))$ having facets $F_{1}, F_{2}, F_{3}$, while $F_{4}:=\left\{x \in P:\left\langle a_{4}, x\right\rangle=0\right\}$ is just the vertex $(0,0)$. Then the point $x:=(-1 / 6,1 / 4)$ satisfies $d_{P}(x)=1 / 3$, however $\left\langle a_{4}, x\right\rangle=1 / 4$. Note that $a_{4}$ is a strict convex combination of $(0,0), a_{1}$ and $a_{2}$. It can be shown that such a behavior cannot occur for canonical rational polytopes in the sense of Definition 2.4 below.

Remark 1.3. As the parameter $s$ varies, the vertices of the adjoint polytopes trace out a skeleton of straight line segments (compare Figure 2 and Lemma 1.12). In computational geometry there are similar constructions such as the medial axis and the straight skeleton [Aichholzer et al. 1995; Eppstein and Erickson 1999], which are of importance in many applications from geography to computer graphics. "Roof constructions" such as $M(P)$ in Proposition 1.14 are also intensively studied in this context (compare Figure 4). The skeleton proposed here is different, since it uses a distance function which is invariant under lattice-preserving affine transformations and not defined in terms of Euclidean distance or angles.

Let us note some elementary properties of polyhedral adjunction:

Proposition 1.4. Let $s \geqslant 0$.

(1) Each facet of $P^{(s)}$ is of the form

$$
F^{(s)}:=\left\{x \in P^{(s)}: d_{F}(x)=s\right\}
$$

for some facet $F$ of $P$.

(2) Assume $P^{(s)}$ has dimension $n$, and let $x \in P^{(s)}$. Then $d_{P^{(s)}}(x)=d_{P}(x)-s$. Moreover, if $x \in \operatorname{int}\left(P^{(s)}\right)$ and $d_{P}(x)=d_{F}(x)$ for a facet $F$ of $P$, then $F^{(s)}$ is a facet of $P^{(s)}$, and $d_{P^{(s)}}(x)=d_{F^{(s)}}(x)$.

(3) Assume $P^{(s)}$ has dimension $n$, and let $r \geqslant 0$. Then

$$
\left(P^{(s)}\right)^{(r)}=P^{(s+r)} .
$$

(4) For $r>0$ we have $r\left(P^{(s)}\right)=(r P)^{(r s)}$.

Proof. Property (1) follows directly from the definition. For (2), we first prove the second statement. Let $x \in \operatorname{int}\left(P^{(s)}\right)$, and let $F$ be a facet of $P$ with $d_{P}(x)=d_{F}(x)$. If we set $\lambda:=s / d_{F}(x)$, we have $\lambda x+(1-\lambda) F \subseteq F^{(s)}$ : all elements $y$ of the 
left-hand side satisfy $d_{F}(y)=s$ and $d_{G}(y) \geqslant s$ for facets $G$ of $P$ other than $F$. This shows that $F^{(s)}$ is indeed $(n-1)$-dimensional.

This also shows that

$$
d_{P}(x)=d_{F}(x)=d_{F^{(s)}}(x)+s \geqslant d_{P^{(s)}}(x)+s .
$$

On the other hand, pick a facet $G$ of $P$ such that $G^{(s)}$ is a facet of $P^{(s)}$ and that $d_{G^{(s)}}(x)=d_{P^{(s)}}(x)$. Then $d_{P}(x) \leqslant d_{G}(x)=d_{G^{(s)}}(x)+s=d_{P^{(s)}}(x)+s$.

Finally, if $x$ sits on the boundary of $P^{(s)}$, then the desired equality reads $0=0$.

Now (3) follows directly from (2), and (4) is immediate from the definition.

The $\mathbb{Q}$-codegree. We now define the invariant we are most interested in. The reciprocal is used to keep the notation consistent with already existing algebrogeometric terminology.

Definition 1.5. We define the $\mathbb{Q}$-codegree of $P$ as

$$
\mu(P):=\left(\sup \left\{s>0: P^{(s)} \neq \varnothing\right\}\right)^{-1},
$$

and the core of $P$ is core $(P):=P^{(1 / \mu(P))}$.

As the following proposition shows, the supremum is actually a maximum. Moreover, since $P$ is a rational polytope, $\mu(P)$ is a positive rational number.

Proposition 1.6. The following quantities coincide:

(1) $\mu(P)$,

(2) $\left(\max \left\{s>0: P^{(s)} \neq \varnothing\right\}\right)^{-1}$,

(3) $\left(\sup \left\{s>0: \operatorname{dim}\left(P^{(s)}\right)=n\right\}\right)^{-1}$,

(4) $\min \left\{p / q>0: p, q \in \mathbb{Z}_{>0},(p P)^{(q)} \neq \varnothing\right\}$,

(5) $\inf \left\{p / q>0: p, q \in \mathbb{Z}_{>0}, \operatorname{dim}\left((p P)^{(q)}\right)=n\right\}$,

(6) $\min \left\{p / q>0: p, q \in \mathbb{Z}_{>0},(p P)^{(q)} \cap \mathbb{Z}^{n} \neq \varnothing\right\}$.

Moreover, core $(P)$ is a rational polytope of dimension $<n$.

Proof. (1), (2), (4) and (6) coincide by Proposition 1.4(4). For the remaining statements, note that for $s>0$, the adjoint polytope $P^{(s)}$ contains a full-dimensional ball if and only if there exists some small $\varepsilon>0$ such that $P^{(s+\varepsilon)} \neq \varnothing$.

The codegree. The $\mathbb{Q}$-codegree is a rational variant of the codegree, which came up in Ehrhart theory of lattice polytopes [Batyrev and Nill 2007]. However, the definition also makes sense for rational polytopes.

Definition 1.7. Let $P$ be a rational polytope. We define the codegree as

$$
\operatorname{cd}(P):=\min \left\{k \in \mathbb{N}_{\geqslant 1}: \operatorname{int}(k P) \cap \mathbb{Z}^{n} \neq \varnothing\right\} .
$$


Lemma 1.8. Let $l$ be the common denominator of all right-hand sides $b_{i}$ given in the inequality description of $P$ as in (*) of Definition 1.1. Then

$$
\operatorname{int}(l P) \cap \mathbb{Z}^{n}=(l P)^{(1)} \cap \mathbb{Z}^{n} .
$$

In particular, $\mu(P) \leqslant l \operatorname{cd}(P)$.

Proof. Let $x \in \operatorname{int}(l P) \cap \mathbb{Z}^{n}$. Then $\mathbb{Z} \ni\left\langle a_{i}, x\right\rangle>l b_{i} \in \mathbb{Z}$ for all $i=1, \ldots, m$. Hence, $\left\langle a_{i}, x\right\rangle \geqslant l b_{i}+1$, as desired. The other inclusion is clear. The last statement follows from Proposition 1.6(6).

Note that for a lattice polytope $P$, we automatically have $l=1$, so

$$
\mu(P) \leqslant \operatorname{cd}(P) \leqslant n+1,
$$

where the last inequality is well-known (take the sum of $n+1$ affinely independent vertices of $P$ ).

The nef value. The third invariant we are going to define is a finite number only if the polytope is not too singular. Let us make this precise.

Definition 1.9. A rational cone $\sigma \subset\left(\mathbb{R}^{n}\right)^{*}$ with primitive generators $v_{1}, \ldots, v_{m}$ in $\left(\mathbb{Z}^{n}\right)^{*}$ is $\mathbb{Q}$-Gorenstein of index $r_{\sigma}$ if there is a primitive point $u_{\sigma} \in \mathbb{Z}^{n}$ with $\left\langle v_{i}, u_{\sigma}\right\rangle=r_{\sigma}$ for all $i$.

The normal fan $\mathcal{N}(P)$ of $P$ is $\mathbb{Q}$-Gorenstein of index $r$ if the maximal cones are $\mathbb{Q}$-Gorenstein and $r=\operatorname{lcm}\left(r_{\sigma}: \sigma \in \mathcal{N}(P)\right)$.

Such a cone/fan is called Gorenstein if the index is 1 . Moreover, we say that $P$ is smooth if for any maximal cone of $\mathcal{N}(P)$ the primitive ray generators form a lattice basis. Clearly, $P$ smooth implies $\mathcal{N}(P)$ Gorenstein.

In other words, $\mathcal{N}(P)$ is $\mathbb{Q}$-Gorenstein if the primitive ray generators of any maximal cone lie in an affine hyperplane and the index equals the least common multiple of the lattice distance of these hyperplanes from the origin. For instance, any simple polytope is $\mathbb{Q}$-Gorenstein because every cone in the normal fan is simplicial.

Definition 1.10. The nef value of $P$ is given as

$$
\tau(P):=\left(\sup \left\{s>0: \mathcal{N}\left(P^{(s)}\right)=\mathcal{N}(P)\right\}\right)^{-1} \in \mathbb{R}_{>0} \cup\{\infty\} .
$$

Note that in contrast to the definition of the $\mathbb{Q}$-codegree, here the supremum is never a maximum.

Definition 1.11. Assume $\mathcal{N}(P)$ is $\mathbb{Q}$-Gorenstein, and $v$ is a vertex of $P$. Assume that in the inequality description of $P$ as in (*) of Definition 1.1, the vertex $v$ satisfies equality precisely for $i \in I$. That is, the normal cone of $v$ is $\sigma=\operatorname{pos}\left(a_{i}: i \in I\right)$. For $s \geqslant 0$, define the point $v(s)$ by $v(s)=v+\left(s / r_{\sigma}\right) u_{\sigma}$, where $u_{\sigma}$ and $r_{\sigma}$ are defined in Definition 1.9. Note that $\left\langle a_{i}, v(s)\right\rangle=b_{i}+s$ for $i \in I$. 


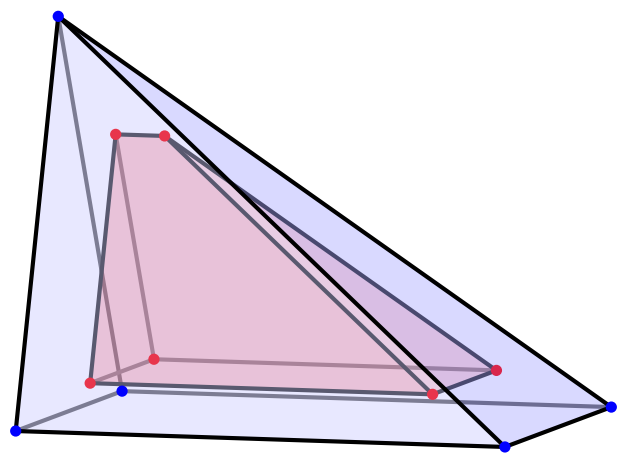

Figure 3. $P^{(1 / 5)} \subseteq P$ for a 3-dimensional lattice polytope $P$.

The following lemma collects various ways to compute the nef value $\tau$ of a polytope, if the normal fan is $\mathbb{Q}$-Gorenstein.

Lemma 1.12. $\mathcal{N}(P)$ is $\mathbb{Q}$-Gorenstein if and only if $\tau(P)<\infty$. Assume this condition holds. Then for $s \in\left[0, \tau(P)^{-1}\right]$ we have $P^{(s)}=\operatorname{conv}(v(s): v$ vertex of $P)$. Consequently, the following quantities coincide:

(1) $\tau(P)^{-1}$,

(2) $\max \left\{s \in \mathbb{Q}_{>0}: v(s) \in P^{(s)}\right.$ for all vertices $v$ of $\left.P\right\}$,

(3) $\min \left\{s \in \mathbb{Q}_{>0}: v(s)=v^{\prime}(s)\right.$ for two different vertices $v$, $v^{\prime}$ of $\left.P\right\}$,

(4) $\min \left\{s \in \mathbb{Q}_{>0}: P^{(s)}\right.$ is combinatorially different from $\left.P\right\}$,

(5) $\max \left\{s \in \mathbb{Q}_{>0}: \mathcal{N}(P)\right.$ refines $\left.\mathcal{N}\left(P^{(s)}\right)\right\}$.

Proof. The first assertion follows by Definition 1.11. Notice that $\mathcal{N}(P)=\mathcal{N}\left(P^{(s)}\right)$ if and only if $v(s) \neq v^{\prime}(s)$ for any two different vertices $v, v^{\prime}$ of $P$. This implies the assertions $(1) \Longleftrightarrow(3) \Longleftrightarrow(4)$. Let now $\xi=\max \left\{s \in \mathbb{Q}_{>0}: v(s) \in P^{(s)}\right\}$. As remarked in Definition 1.11 it is $\tau(P)^{-1} \leqslant \xi$. On the other hand the existence of an $s \in \mathbb{Q}$ such that $\xi<s<\tau(P)^{-1}$ would lead to a contradiction. In fact it would imply that $\mathcal{N}(P)=\mathcal{N}\left(P^{(s)}\right)$ and the existence of a vertex $v \in P$ for which $v(s) \notin P^{(s)}$. This proves $(1) \Longleftrightarrow(2) \Longleftrightarrow(5)$.

Figure 3 shows a three-dimensional lattice polytope $P$ whose normal fan is not $\mathbb{Q}$-Gorenstein $(\tau(P)=\infty)$. Note that $P$ has 5 vertices, while the adjoint polytope $P^{(c)}$ (for $0<c<1 / \mu(P)$ ) has 6 vertices.

By definition, we have $\mu(P) \leqslant \tau(P)$. We also want to compare the codegree and the nef value.

Proposition 1.13. Let $P$ be a lattice polytope with $\mathbb{Q}$-Gorenstein normal fan of index $r$. If $s \geqslant r \tau(P)$ is an integer, then $(s P)^{(r)}$ is a lattice polytope. In particular,

$$
\operatorname{cd}(P)-1<r \tau(P) \text {. }
$$


Proof. By Lemma 1.12 every vertex of $P^{(r / s)}$ is of the form $v\left(\frac{r}{s}\right)=v+\frac{r}{r_{\sigma} s} u_{\sigma}$ for some vertex $v$ of $P$. Hence, every vertex of $(s P)^{(r)}$ is given as $s v\left(\frac{r}{s}\right)=s v+\frac{r}{r_{\sigma}} u_{\sigma}$, a lattice point. For the last statement it suffices to observe that $(\operatorname{cd}(P)-1) P$ does not have interior lattice points.

The "mountain" and $\mathbb{Q}$-normality. We now give a graphical description of the nef value and the $\mathbb{Q}$-codegree, one that provides an efficient way to compute these invariants. Let the mountain $M(P) \subseteq \mathbb{R}^{n+1}$ be defined as

$$
M(P):=\left\{(x, s): x \in P, 0 \leqslant s \leqslant d_{P}(x)\right\} .
$$

Proposition 1.14. Assume that $P$ has an inequality description as in formula (*) of Definition 1.1. Then

$$
M(P)=\left\{(x, s) \in \mathbb{R}^{n+1}:(A \mid-\mathbb{1})(x, s)^{\mathrm{T}} \geqslant b, s \geqslant 0\right\} .
$$

Therefore, $M(P)$ is a rational polytope with $M(P) \cap \mathbb{R}^{n} \times\left\{s_{0}\right\}=P^{\left(s_{0}\right)} \times\left\{s_{0}\right\}$. Moreover,

$$
\mu(P)^{-1}=\max (s: \text { there is a vertex of } M(P) \text { with last coordinate } s) .
$$

If $\mathcal{N}(P)$ is $\mathbb{Q}$-Gorenstein, then

$$
\tau(P)^{-1}=\min (s>0: \text { there is a vertex of } M(P) \text { with last coordinate } s) .
$$

Proof. Set $q:=\mu(P)^{-1}$. By Proposition 1.6(2), we have $q=\max \left\{s>0: P^{(s)} \neq \varnothing\right\}$. By the definition of $P^{(s)}$, this is the maximal positive $s$ such that there is an $x \in P$ which satisfies $d_{F}(x) \geqslant s$ for all facets $F$ of $P$. This shows (1).

Let us prove (2). Suppose $\mathcal{N}(P)$ is $\mathbb{Q}$-Gorenstein, and abbreviate $t:=\tau(P)^{-1}$. For every vertex $v$ of $P$ and $s>0$ define $v(s)$ as in Definition 1.11. At every vertex $(v, 0)$ of the bottom facet $P \times\{0\}$ of $M(P)$ there is a unique upwards edge towards $(v(s), s)$ for small $s$. By Lemma 1.12(3) there are two vertices $v, v^{\prime}$ of $P$ so that $v(t)=v^{\prime}(t)$. The corresponding point $(v(t), t)=\left(v^{\prime}(t), t\right)$ in $M(P)$ is a vertex, as it is incident to at least two edges.

Let us consider the example given on the right-hand side of Figure 1, and take a look at its mountain - see Figure 4. The height of the mountain equals the reciprocal of the $\mathbb{Q}$-codegree, while the height of the first nontrivial vertex is the reciprocal of the nef value.

This motivates the following definition (see [Dickenstein et al. 2009]).

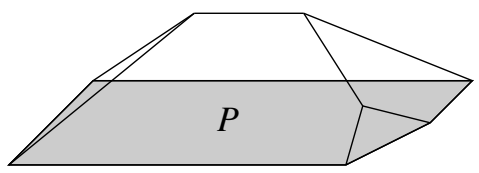

Figure 4. The lattice distance mountain $M(P)$. 


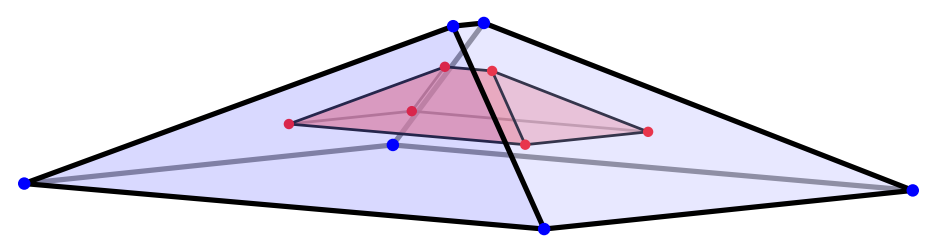

Figure 5. $P^{(4)} \subseteq P$ for a 3-dimensional lattice polytope $P$.

Definition 1.15. We say that $P$ is $\mathbb{Q}$-normal if $\mu(P)=\tau(P)$.

To get the correct intuition for this notion, let us note that $P$ is $\mathbb{Q}$-normal if and only if all vertices of $P$ survive under polyhedral adjunction (as long as the adjoint polytope is full-dimensional). For $n \geqslant 3$ it is not enough that all facets of $P$ survive, as Figure 5 illustrates (where $\tau(P)^{-1}=2, \mu(P)^{-1}=6$ and core $(P)$ is an interval).

\section{Natural projections}

Throughout let $P \subseteq \mathbb{R}^{n}$ be an $n$-dimensional rational polytope.

The core and the natural projection. Recall that $\operatorname{core}(P):=P^{(1 / \mu(P))}$ is a rational polytope of dimension $<n$.

Definition 2.1. Let $K(P)$ be the linear space parallel to aff(core $(P))$. We call $\pi_{P}: \mathbb{R}^{n} \rightarrow \mathbb{R}^{n} / K(P)$ the natural projection associated with $P$.

Lemma 2.2. Let $x \in \operatorname{relint}(\operatorname{core}(P))$. Let us denote by $F_{1}, \ldots, F_{t}$ the facets of $P$ with $d_{F_{i}}(x)=\mu(P)^{-1}$. Then their primitive inner normals $a_{1}, \ldots, a_{t}$ positively span the linear subspace $K(P)^{\perp}$.

Moreover, if $\operatorname{core}(P)=\{x\}$, then

$$
\left\{y \in \mathbb{R}^{n}: d_{F_{i}}(y) \geqslant 0 \text { for all } i=1, \ldots, t\right\}
$$

is a rational polytope containing $P$.

Proof. We set $s:=\mu(P)^{-1}$. Let $i \in\{1, \ldots, t\}$. Since $d_{F_{i}}(x)=s$ and $x \in \operatorname{relint}\left(P^{(s)}\right)$, we have $d_{F_{i}}(y)=s$ for all $y \in P^{(s)}$. This shows $C:=\operatorname{pos}\left(a_{1}, \ldots, a_{t}\right) \subseteq K(P)^{\perp}$. Assume that this inclusion were strict. Then there exists some $v \in \mathbb{R}^{n}$ such that $\langle v, C\rangle \geqslant 0$ and $v$ does not vanish on the linear subspace $K(P)^{\perp}$. In particular, for any $i \in\{1, \ldots, t\}$ one gets $\left\langle v, a_{i}\right\rangle \geqslant 0$, so $d_{F_{i}}(x+\varepsilon v) \geqslant d_{F_{i}}(x)=s$ for any $\varepsilon>0$. Moreover, if we choose $\varepsilon$ small enough, then $d_{G}(x+\varepsilon v) \approx d_{G}(x)>s$ for any other facet $G$ of $P$. Hence, $x+\varepsilon v \in P^{(s)}$. But this means $v \in K(P)$, and $v$ must vanish on $K(P)^{\perp}$, a contradiction.

Finally, notice that if $P^{(s)}=\{x\}$, then $a_{1}, \ldots, a_{t}$ positively span $\left(\mathbb{R}^{n}\right)^{*}$. In particular, $\operatorname{conv}\left(a_{1}, \ldots, a_{t}\right)$ contains a small full-dimensional ball around the origin. Dually, $\left\{y \in \mathbb{R}^{n}:\left\langle a_{i}, y\right\rangle \geqslant b_{i}, i=1, \ldots, t\right\}$ is contained in a large ball. Hence, it is a bounded rational polyhedron, thus a rational polytope. 

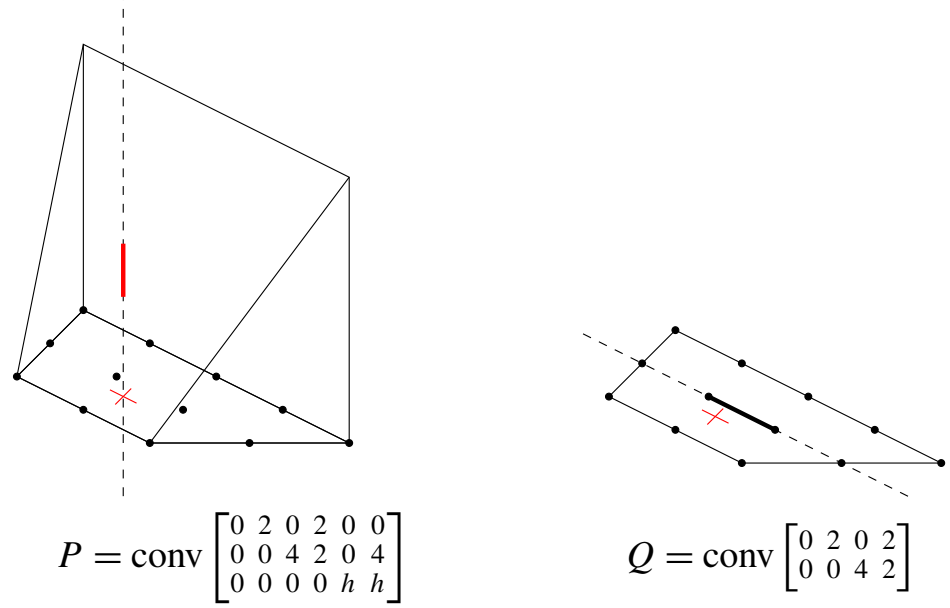

Figure 6. The $\mathbb{Q}$-codegree projection $\pi_{P}: P \rightarrow Q$.

The $\mathbb{Q}$-codegree under natural projections. We begin with a key observation.

Proposition 2.3. The image $Q:=\pi_{P}(P)$ of the natural projection of $P$ is a rational polytope satisfying $\mu(Q) \geqslant \mu(P)$. Moreover, if $\mu(Q)=\mu(P)$, then $\operatorname{core}(Q)$ is the point $\pi_{P}(\operatorname{core}(P))$.

Proof. Let $t, x, F_{i}, a_{i}$ as in Lemma 2.2 and $s:=\mu(P)^{-1}$. $Q$ is a rational polytope with respect to the lattice $L:=\mathbb{Z}^{n} /\left(K(P) \cap \mathbb{Z}^{n}\right)$. The dual lattice of $L$ is $\left(\mathbb{Z}^{n}\right)^{*} \cap K(P)^{\perp}$. In particular, any $a_{i}$ for $i \in\{1, \ldots, t\}$ is still a primitive normal vector of a facet of $Q$. In particular, $Q^{(s)} \subseteq \pi_{P}\left(P^{(s)}\right)=\left\{\pi_{P}(x)\right\}$. Therefore, $\mu(Q)^{-1} \leqslant s$.

The example in Figure 6 shows that this projection can be quite peculiar. The dashed lines are the affine hulls along which we are projecting, while the fat line segments are the cores of $P$ and $Q$. On the left side we only drew the lattice points on the bottom face for clarity. Here, $\pi_{P}$ projects onto the bottom face $Q$. If we assume that the height $h$ of $P$ is large enough, then the adjoint polytope core $(P)$ is a line segment projecting onto the point $x=\left(\frac{4}{3}, \frac{4}{3}, 0\right)$ marked on the bottom. Note that this point doesn't even lie in the line segment core $(Q)$. Essentially, the reason for this behavior is that the preimage of one of the two facets of $Q$ defining the affine hull of $\operatorname{core}(Q)$ is not a facet of $P$. Moreover, $\mu(Q)=1>\frac{3}{4}=\mu(P)$.

\section{Projections of $\alpha$-canonical polytopes.}

Definition 2.4. Let $\sigma$ be a rational cone with primitive generators $v_{1}, \ldots, v_{m}$. Then the height function associated with $\sigma$ is the piecewise linear function

$$
\mathrm{ht}_{\sigma}(x):=\max \left\{\sum_{i=1}^{m} \lambda_{i}: \lambda_{i} \geqslant 0 \text { for } i=1, \ldots, m, \sum_{i=1}^{m} \lambda_{i} v_{i}=x\right\}
$$


on $\sigma$. For $\alpha>0$, we say that $\sigma$ is $\alpha$-canonical if $\mathrm{ht}_{\sigma}(x) \geqslant \alpha$ for every nonzero $x \in \sigma \cap \mathbb{Z}^{n}$. A 1-canonical cone is said to be canonical.

A rational polytope is $(\alpha-)$ canonical if all cones of its normal fan are.

This is a generalization to the non- $\mathbb{Q}$-Gorenstein case of canonical singularities in algebraic geometry. Note that a $\mathbb{Q}$-Gorenstein cone of index $r$ is $(1 / r)$-canonical. In particular, rational polytopes with Gorenstein normal fan are canonical.

Lemma 2.5. Let $\pi: P \rightarrow Q$ be a polytope projection, and assume $P$ is $\alpha$-canonical. Then $\alpha d_{P}(x) \leqslant d_{Q}(\pi(x))$ for all $x \in P$.

Proof. Let $\langle a, \cdot\rangle \geqslant b$ be a facet of $Q$ realizing $d_{Q}(\pi(x))$. That is, $\langle a, \pi(x)\rangle=$ $b+d_{Q}(\pi(x))$. Then the integral linear functional $\pi^{*} a$ belongs to some cone $\sigma \in \mathcal{N}(P)$ with primitive generators $a_{1}, \ldots, a_{m}$. Write $\pi^{*} a=\sum_{i=1}^{m} \lambda_{i} a_{i}$ with $\lambda_{i} \geqslant 0$ for $i=1, \ldots, m$ and $\sum_{i=1}^{m} \lambda_{i}=\mathrm{ht}_{\sigma}\left(\pi^{*} a\right)$. Then $b=\sum_{i=1}^{m} \lambda_{i} b_{i}$, and $\sum_{i=1}^{m} \lambda_{i} \geqslant \alpha$. Thus

$$
\begin{aligned}
d_{Q}(\pi(x)) & =\langle a, \pi(x)\rangle-b=\left\langle\pi^{*} a, x\right\rangle-b \\
& =\sum_{i=1}^{m} \lambda_{i}\left(\left\langle a_{i}, x\right\rangle-b_{i}\right) \geqslant \sum_{i=1}^{m} \lambda_{i} d_{P}(x) \geqslant \alpha d_{P}(x) .
\end{aligned}
$$

Corollary 2.6. Let $\pi: P \rightarrow Q$ be a polytope projection, and assume $P$ is $\alpha$ canonical. Then $\mu(P) \geqslant \alpha \mu(Q)$.

In particular, if $P$ is canonical, then $\mu(P) \geqslant \mu(Q)$.

This shows that for canonical polytopes the natural projection in Proposition 2.3 is $\mathbb{Q}$-codegree preserving! In particular, the polytope $Q$ has the nice property that $\operatorname{core}(Q)$ is a point.

Example 2.7. Unfortunately, it is in general not true that being $\alpha$-canonical is preserved under the natural projection, as can be seen from the following example. Consider the polytope

$$
P=\operatorname{conv}\left[\begin{array}{rrrrrrrrr}
14 & 8 & 0 & -8 & 14 & 0 & 0 & -14 & -14 \\
7 & 1 & 0 & 1 & 7 & 21 & 21 & 7 & 7 \\
-21 & -3 & 0 & 3 & 21 & 21 & -21 & 21 & -21
\end{array}\right]
$$

This is a three-dimensional lattice polytope. Its core has the vertices $(0,7,7)$ and $(0,7,-7)$, so the natural projection $\pi$ maps onto a two-dimensional lattice polytope by projecting onto the first two coordinates. The projection is

$$
\pi(P)=\operatorname{conv}\left[\begin{array}{rrrrrr}
14 & 8 & 0 & -8 & 0 & -14 \\
7 & 1 & 0 & 1 & 21 & 7
\end{array}\right]
$$

All but one normal cone of $P$ is canonical. The exception is the normal cone at the origin. Its primitive rays are $(-1,-5,-1),(1,-5,1),(0,-3,-1)$ and $(0,-3,1)$. The ray $(0,-1,0)$ is in the cone, and its height is $\frac{1}{3}$. So $P$ is $\frac{1}{3}$-canonical. The normal cones of the natural projection $Q$ are again canonical with one exception. 
The normal cone at the origin is generated by the rays $(1,-8)$ and $(-1,-8)$. It contains the ray $(0,-1)$, so $Q$ is only $\frac{1}{8}$-canonical. The computations were done with polymake [Joswig et al. 2009].

\section{$\mathbb{Q}$-normality under natural projections.}

Proposition 2.8. Let $P$ be $\mathbb{Q}$-normal. Then its image $Q$ under the natural projection is $\mathbb{Q}$-normal, its core is the point $\operatorname{core}(Q)=\pi_{P}(\operatorname{core}(P))$, and $\mu(Q)=\mu(P)$. Moreover, if $P$ is $\alpha$-canonical, then $Q$ is $\alpha$-canonical.

Proof. If $P$ is $\mathbb{Q}$-normal, then the normal fan of $P$ refines the normal fan of core $(P)=P^{(1 / \tau(P))}$. In particular, the face $K(P)^{\perp}$ of $\mathcal{N}(\operatorname{core}(P))$ is a union of faces of $\mathcal{N}(P)$. Therefore, being $\alpha$-canonical is preserved. On the other hand, $\mathcal{N}(Q)=\mathcal{N}(P) \cap K(P)^{\perp}$ for any polytope projection $P \rightarrow Q$. That means that every facet $F$ of $Q$ lifts to a facet $\pi_{P}^{*} F$ of $P$. Together with $d_{F}\left(\pi_{P}(x)\right)=d_{\pi_{P}^{*} F}(x)$ (for $x \in P)$ this implies $Q^{(s)}=\pi\left(P^{(s)}\right)$ for any $s \geqslant 0$. This yields the statements.

If a rational polytope is $\mathbb{Q}$-normal and its core is a point, then the generators of its normal fan form the vertex set of a lattice polytope. Such a fan corresponds to a toric Fano variety; see, e.g., [Debarre 2003; Nill 2005].

\section{Cayley decompositions}

Throughout let $P \subseteq \mathbb{R}^{n}$ be an $n$-dimensional lattice polytope.

Lattice width, Cayley polytopes and codegree. We recall that the lattice width of a polytope $P$ is defined as the minimum of $\max _{x \in P}\langle u, x\rangle-\min _{x \in P}\langle u, x\rangle$ over all nonzero integer linear forms $u$. We are interested in lattice polytopes of lattice width one, which we also call (nontrivial) Cayley polytopes or Cayley polytopes of length $\geqslant 2$.

Definition 3.1. Given lattice polytopes $P_{0}, \ldots, P_{t}$ in $\mathbb{R}^{k}$, the Cayley sum $P_{0} * \cdots * P_{t}$ is defined to be the convex hull of $\left(P_{0} \times 0\right) \cup\left(P_{1} \times e_{1}\right) \cup \cdots \cup\left(P_{t} \times e_{t}\right)$ in $\mathbb{R}^{k} \times \mathbb{R}^{t}$ for the standard basis $e_{1}, \ldots, e_{t}$ of $\mathbb{R}^{t}$.

We say that $P \subseteq \mathbb{R}^{n}$ is a Cayley polytope of length $t+1$ if there exists an affine lattice basis of $\mathbb{Z}^{n} \cong \mathbb{Z}^{k} \times \mathbb{Z}^{t}$ identifying $P$ with the Cayley sum $P_{0} * \cdots * P_{t}$ for some lattice polytopes $P_{0}, \ldots, P_{t}$ in $\mathbb{R}^{k}$.

This definition can be reformulated [Batyrev and Nill 2008, Proposition 2.3].

Lemma 3.2. Let $\sigma \subseteq \mathbb{R}^{n+1}$ be the cone spanned by $P \times 1$. Then the following statements are equivalent:

(1) $P$ is a Cayley polytope $P_{0} * \cdots * P_{t}$ of length $t+1$.

(2) There is a lattice projection P onto a unimodular $t$-simplex. 
(3) There are nonzero $x_{1}, \ldots, x_{t+1} \in \sigma^{\vee} \cap\left(\mathbb{Z}^{n+1}\right)^{*}$ such that

$$
x_{1}+\cdots+x_{t+1}=e_{n+1} .
$$

Since the $t$-th multiple of a unimodular $t$-simplex contains no interior lattice points, we conclude from Lemma 3.2(2) that

$$
\operatorname{cd}\left(P_{0} * \cdots * P_{t}\right) \geqslant t+1 .
$$

Conversely, Conjecture 2 states that having large codegree implies being a Cayley polytope. To get the reader acquainted with Conjecture 2, we include a simple observation.

Lemma 3.3. If $\operatorname{cd}(P)>\lceil(n+1) / 2\rceil$, then through every vertex there is an edge whose only lattice points are its two vertices.

Proof. Assume otherwise. Then there exists an injective lattice homomorphism $f$ mapping $2 \Delta_{n} \rightarrow P$. Therefore, Stanley's monotonicity theorem [Stanley 1993; Batyrev and Nill 2007] yields $n+1-\operatorname{cd}\left(f\left(2 \Delta_{n}\right)\right) \leqslant n+1-\operatorname{cd}(P)$, hence $\operatorname{cd}(P) \leqslant \operatorname{cd}\left(f\left(2 \Delta_{n}\right)\right) \leqslant \operatorname{cd}\left(2 \Delta_{n}\right)=\lceil(n+1) / 2\rceil$. This yields a contradiction to our assumption.

The decomposition theorem. Let $P, P^{\prime}$ be $n$-dimensional lattice polytopes. We will say that $P$ and $P^{\prime}$ are unimodularly equivalent $\left(P \cong P^{\prime}\right)$ if there exists an affine lattice automorphism of $\mathbb{Z}^{n}$ mapping the vertices of $P$ onto the vertices of $P^{\prime}$. It is a well-known result (see, for example, [Batyrev and Nill 2007]) that $P \cong \Delta_{n}$ if and only if $\operatorname{cd}(P)=n+1$. Since $\mu(P) \leqslant \operatorname{cd}(P) \leqslant n+1$ and $\mu\left(\Delta_{n}\right)=n+1$, we deduce that $P \cong \Delta_{n}$ if and only if $\mu(P)=n+1$.

We next prove a general structure result on lattice polytopes of high $\mathbb{Q}$-codegree. We set

$$
d(P):= \begin{cases}2(n-\lfloor\mu(P)\rfloor) & \text { if } \mu(P) \notin \mathbb{N}, \\ 2(n-\mu(P))+1 & \text { if } \mu(P) \in \mathbb{N} .\end{cases}
$$

If we exclude the special situation $P \cong \Delta_{n}$, we have $1 \leqslant d(P)<2(n+1-\mu(P))$.

Theorem 3.4. Let $P$ be an $n$-dimensional lattice polytope with $P ¥ \Delta_{n}$. If $n>d(P)$, then $P$ is a Cayley sum of lattice polytopes in $\mathbb{R}^{m}$ with $m \leqslant d(P)$.

For the proof we recall the following folklore result.

Lemma 3.5. Let $P \subseteq \mathbb{R}^{n}$ be an $n$-dimensional lattice polytope. Let

$$
z \in \operatorname{pos}(P \times\{1\}) \cap \mathbb{Z}^{n+1} \text {. }
$$

Then there exist (not necessarily different) vertices $v_{1}, \ldots, v_{g}$ of $P$ and a lattice point $p \in(j P) \cap \mathbb{Z}^{n}$ with

$$
z=\left(v_{1}, 1\right)+\cdots+\left(v_{g}, 1\right)+(p, j)
$$




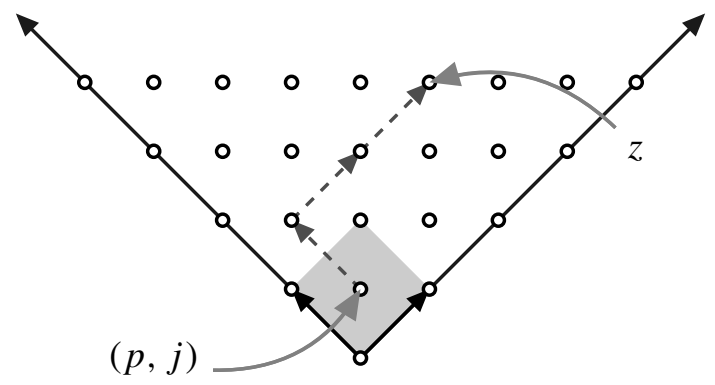

Figure 7. Decomposing $z$ in the proof of Lemma 3.5.

such that $(p, j)=(0,0)$ or $1 \leqslant j \leqslant n+1-\operatorname{cd}(P)$.

Proof. There exists an $m$-dimensional simplex $S$ in $P$ with vertices $v_{1}, \ldots, v_{m+1}$ in the vertex set of $P$ such that $z \in \operatorname{pos}\left(\left(v_{1}, 1\right), \ldots,\left(v_{m+1}, 1\right)\right)$. We can write

$$
z=\sum_{i=1}^{m+1} k_{i}\left(v_{i}, 1\right)+\sum_{i=1}^{m+1} \lambda_{i}\left(v_{i}, 1\right) \quad \text { for } k_{i} \in \mathbb{N} \text { and } \lambda_{i} \in[0,1) .
$$

See also Figure 7. The lattice point $\sum_{i=1}^{m+1} \lambda_{i}\left(v_{i}, 1\right)$ is an element of the fundamental parallelepiped of the simplex $S$. By [Beck and Robins 2007, Corollary 3.11], its height $j$ equals at most the degree of the so-called Ehrhart $h^{*}$-polynomial. EhrhartMacdonald reciprocity implies that this degree is given by $m+1-\operatorname{cd}(S)$. We refer to [Batyrev and Nill 2007] for more details. Now, the result follows from $j \leqslant m+1-\operatorname{cd}(S) \leqslant n+1-\operatorname{cd}(P)$ by Stanley's monotonicity theorem [1993].

Proof of Theorem 3.4. By successive application of Proposition 2.3, we can find a lattice projection $P \rightarrow Q$ with $\operatorname{dim}(Q)=n^{\prime} \leqslant n$ such that $\mu(P) \leqslant \mu(Q)$ and $Q^{(s)}=\{x\}$ for $s:=\mu(Q)^{-1}$. By observing that $d(Q)+\left(n-n^{\prime}\right) \leqslant d(P)$, we see that $d(P)<n$ implies $d(Q)<n^{\prime}$ and, moreover, if the desired statement holds for $Q$, then it also holds for $P$. Hence, we may assume that $s=\mu(P)^{-1}$ and $P^{(s)}=\{x\}$.

By Lemma 2.2,P is contained in a rational polytope $\tilde{P}$ with $s=\mu(\tilde{P})^{-1}$ and $\tilde{P}^{(s)}=\{x\}$ so that all facets of $\tilde{P}$ have distance $s$ from $x$. Let $\sigma \subseteq \tilde{\sigma} \subseteq \mathbb{R}^{n+1}$ be the (full-dimensional, pointed) cones over $P \times\{1\} \subseteq \tilde{P} \times\{1\}$, and let $u \in\left(\mathbb{R}^{n+1}\right)^{*}$ be the last coordinate functional. As $u$ evaluates positively on all vertices of $\tilde{P} \times\{1\}$, we have $u \in \operatorname{int} \tilde{\sigma}^{\vee} \subseteq$ int $\sigma^{\vee}$. Let us define the lattice polytope

$$
R:=\operatorname{conv}(\{0\} \cup\{\eta: \eta \text { primitive facet normal of } \tilde{\sigma}\}) \subseteq\left(\mathbb{R}^{n+1}\right)^{*} .
$$

In order to invoke Lemma 3.2(3), we will show that $R$ has high codegree so that $u$ can be decomposed into a sum of many lattice points in $\tilde{\sigma}^{\vee} \subseteq \sigma^{\vee}$ by Lemma 3.5. 
To this end, observe that $\langle\eta,(x, 1)\rangle=s$ for every primitive facet normal $\eta$ of $\tilde{\sigma}$, so that $R$ is an $(n+1)$-dimensional pyramid with apex 0 :

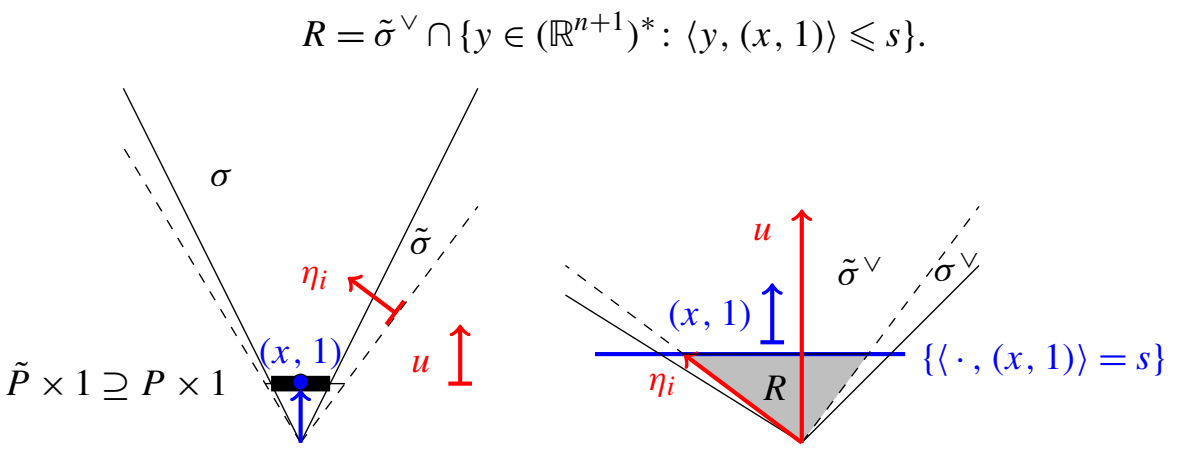

Let us bound the height of an interior lattice point of $\tilde{\sigma}^{\vee}$. Assume there is some $y \in$ int $\tilde{\sigma}^{\vee} \cap\left(\mathbb{Z}^{n+1}\right)^{*}$ such that $\langle y,(x, 1)\rangle<1$. Because $x \in P$ is a convex combination of vertices there is some vertex $w \in P \times\{1\}$ such that $\langle y, w\rangle<1$. However, $y \in \operatorname{int} \tilde{\sigma}^{\vee} \subseteq$ int $\sigma^{\vee}$ implies $0<\langle y, w\rangle$. This contradicts $\langle y, w\rangle \in \mathbb{Z}$. Now, $\langle\cdot,(x, 1)\rangle \leqslant s$ is a valid inequality for $R$, and by the above, $\operatorname{int}(k R) \cap\left(\mathbb{Z}^{n+1}\right)^{*}=\varnothing$ for $k \leqslant s^{-1}=\mu(P)$.

On the other hand, $u$ is a lattice point in int $\tilde{\sigma}^{\vee}$ with $\langle u,(x, 1)\rangle=1$. So $u$ is in $\operatorname{int}(k R) \cap\left(\mathbb{Z}^{n+1}\right)^{*}$ for $k>\mu(P)$. Hence, $r:=\operatorname{cd}(R)=\lfloor\mu(P)\rfloor+1$.

From Lemma 3.5 applied to $R$ and $(u, r) \in \operatorname{pos}(R \times\{1\}) \cap\left(\mathbb{Z}^{n+2}\right)^{*}$ we conclude that

$$
(u, r)=k(0,1)+\left(\eta_{1}, 1\right)+\cdots+\left(\eta_{g}, 1\right)+(p, j)
$$

for a natural number $k$, for (not necessarily different) nonzero vertices $\eta_{1}, \ldots, \eta_{g}$ of $R$ and for a lattice point $p \in(j R) \cap\left(\mathbb{Z}^{n+1}\right)^{*}$ with the property that $(p, j)=(0,0)$ or $1 \leqslant j \leqslant n+2-r$.

From $u \notin(r-2) R$ and $(u, r-2)=(k-2)(0,1)+\left(\eta_{1}, 1\right)+\cdots+\left(\eta_{g}, 1\right)+(p, j)$, we conclude that $k-2<0$, that is, $k \in\{0,1\}$. Further, if $k=1$, then $u$ is in $(r-1) R \backslash \operatorname{int}((r-1) R)$ so that $1=\langle u,(x, 1)\rangle=(r-1) s$, that is, $\mu(P) \in \mathbb{Z}$.

Let us first consider the case $k=0$. Since $u \in \operatorname{int}(r R)$, we observe that

$$
(u, r) \notin \operatorname{pos}\left(\left(\eta_{1}, 1\right), \ldots,\left(\eta_{g}, 1\right)\right),
$$

thus $(p, j) \neq(0,0)$. Therefore, $r=g+j$, and $u$ splits into a sum of at least $g+1 \geqslant r+1-(n+2-r)=2\lfloor\mu(P)\rfloor-n+1$ nonzero lattice vectors in $\tilde{\sigma}^{\vee}$. Hence, Lemma 3.2(3) yields that $P$ is a Cayley polytope of lattice polytopes in $\mathbb{R}^{m}$ with $m \leqslant n+1-(g+1) \leqslant 2(n-\lfloor\mu(P)\rfloor)$.

It remains to deal with the case $k=1$. Here, we have already observed that $\mu(P) \in \mathbb{Z}$. If $(p, j)=(0,0)$, then $u$ splits into a sum of at least $g+1=r$ nonzero lattice points in $\tilde{\sigma}^{\vee}$, so Lemma 3.2(3) yields that $P$ is the Cayley polytope of lattice 
polytopes in $\mathbb{R}^{m}$ with $m \leqslant n+1-(g+1) \leqslant n+1-\mu(P)$. Finally, if $(p, j) \neq(0,0)$, then $r=g+1+j$, so we again deduce from Lemma 3.2(3) that $P$ is the Cayley polytope of $g+1=r-j \geqslant r-(n+2-r)=2 r-n-2$ lattice polytopes in an ambient space of dimension $n+1-(2 r-n-2)=2(n-\mu(P))+1$.

Remark 3.6. Statement and proof of Theorem 3.4 generalize Theorem 3.1 in [Haase et al. 2009], which proves Conjecture 2 in the case of Gorenstein polytopes. A Gorenstein polytope $P$ with codegree $c$ can be characterized by the property that $P$ is a $\mathbb{Q}$-normal lattice polytope with $(c P)^{(1)}$ being a lattice point.

Corollary 3.7. Let $P$ be an n-dimensional lattice polytope. If $n$ is odd and $\mu(P)>$ $(n+1) / 2$, or if $n$ is even and $\mu(P) \geqslant(n+2) / 2$, then $P$ is a Cayley polytope.

There is no obvious analogue for rational polytopes. For instance, for $\varepsilon>0$, the $\mathbb{Q}$-codegree of $(1+\varepsilon) \Delta_{n}$ equals $(n+1) /(1+\varepsilon)$, so it gets arbitrarily close to $n+1$, however its lattice width is always strictly larger than one.

Theorem 3.4 proves Conjecture 2 if $\lceil\mu(P)\rceil=\operatorname{cd}(P)$. Therefore, we get the following new result using Proposition 1.13.

Corollary 3.8. Conjecture 2 holds if $\mathcal{N}(P)$ is Gorenstein and $P$ is $\mathbb{Q}$-normal.

If $P$ is smooth with $\operatorname{cd}(P)>(n+2) / 2$, then it was shown in [Dickenstein et al. 2009; Dickenstein and Nill 2010] that $P \cong P_{0} * \cdots * P_{t}$, where $t+1=\operatorname{cd}(P)=\mu(P)$, and $P_{0}, \ldots, P_{t}$ have the same normal fan. The proof relies on algebraic geometry; no purely combinatorial proof is known.

A sharper conjecture. We conjecture that in Corollary 3.7 the condition $\mu(P)>$ $(n+1) / 2$ should also be sufficient in even dimension. This is motivated by an open question in algebraic geometry - see Remark 4.10. We can prove this conjecture in the case of lattice simplices.

Proposition 3.9. Let $P \subseteq \mathbb{R}^{n}$ be an $n$-dimensional rational simplex. Let $a_{i}$ be the lattice distance of the $i$-th vertex of $P$ from the facet of $P$ not containing the vertex. Then

$$
\tau(P)=\mu(P)=\sum_{i=0}^{n} \frac{1}{a_{i}} .
$$

Proof. Let $x$ be the unique point that has the same lattice distance $s$ from each facet. Then $\tau(P)^{-1}=\mu(P)^{-1}=s$. Fix a basis $\left\{e_{0}, \ldots, e_{n}\right\}$ for $\mathbb{R}^{n+1}$ and consider the affine isomorphism

$$
P \rightarrow \operatorname{conv}\left(a_{0} e_{0}, \ldots, a_{n} e_{n}\right)=\left\{y \in \mathbb{R}_{\geqslant 0}^{n+1}: \sum_{i=0}^{n} \frac{y_{i}}{a_{i}}=1\right\} \subset \mathbb{R}^{n+1}
$$

given by $y \mapsto\left(d_{F_{0}}(y), \ldots, d_{F_{n}}(y)\right)$. The point $x$ is mapped to $c:=(s, \ldots, s)$, so $1 / s=\sum_{i=0}^{n} 1 / a_{i}$. 
Corollary 3.10. Let $P \subseteq \mathbb{R}^{n}$ be an $n$-dimensional lattice simplex.

(1) If $\mu(P)>(n+1) / 2\left(\right.$ or $\mu(P)=(n+1) / 2$ and $a_{i} \neq 2$ for some $\left.i\right)$, then $P$ is $a$ lattice pyramid.

(2) If $\mu(P) \geqslant(n+1) / 2$ and $P \nsucceq 2 \Delta_{n}$, then $P$ has lattice width one.

Proof. Assume that $P$ is not a lattice pyramid. Then $a_{i} \geqslant 2$ for all $i=0, \ldots, n$. Hence,

$$
\mu(P)=\sum_{i=0}^{n} \frac{1}{a_{i}} \leqslant \frac{n+1}{2} .
$$

This proves (1). For (2), let us assume that $a_{i}=2$ for all $i=0, \ldots, n$. We consider the injective affine map

$$
\mathbb{R}^{n} \rightarrow \mathbb{R}^{n}, \quad y \mapsto\left(d_{F_{1}(y)}, \ldots, d_{F_{n}}(y)\right) .
$$

Note that the image of $P$ is $2 \Delta_{n}=\operatorname{conv}\left(0,2 e_{1}, \ldots, 2 e_{n}\right)$. Let us denote the image of $\mathbb{Z}^{n}$ by $\Lambda$. It satisfies $2 \mathbb{Z}^{n} \subseteq \Lambda \subseteq \mathbb{Z}^{n}$. If $\Lambda=\mathbb{Z}^{n}$, then $P \cong 2 \Delta_{n}$. Hence, our assumption yields that the reduction modulo 2 is a proper linear subspace $\Lambda / 2 \mathbb{Z}^{n} \subset(\mathbb{Z} / 2 \mathbb{Z})^{n}$. Therefore, it must satisfy an equation $\sum_{i \in I} x_{i} \equiv 0 \bmod 2$ for some subset $\varnothing \neq I \subseteq\{1, \ldots, n\}$. The linear functional $\frac{1}{2}\left(\sum_{i \in I} x_{i}\right)$ defines an element $\lambda \in \Lambda^{*}$ such that $\lambda\left(2 e_{i}\right)=1$ if $i \in I$ and 0 otherwise. Hence, $P$ has lattice width one in the direction of the pullback of $\lambda$.

Example 3.11. It is tempting to guess that $\mu(P)=(n+1) / 2$ and $a_{i}=2$ for all $i$ implies that $P \cong 2 \Delta_{n}$. However, here is another example:

$$
\text { conv }\left[\begin{array}{llll}
0 & 0 & 1 & 1 \\
0 & 1 & 0 & 1 \\
0 & 1 & 1 & 0
\end{array}\right] \text {. }
$$

A corresponding result for the codegree was proven in [Nill 2008] where it is shown that a lattice $n$-simplex is a lattice pyramid if $\operatorname{cd}(P) \geqslant \frac{3}{4}(n+1)$. Let us stress that Conjecture 2 is still open for lattice simplices.

\section{Adjunction theory of toric varieties}

In this section, we explain the connection between the previous combinatorial results and the adjunction theory of toric varieties.

General notation and definitions. Let $X$ be a normal projective algebraic variety of dimension $n$ with canonical class $K_{X}$ defined over the complex numbers. We assume throughout that $X$ is $\mathbb{Q}$-Gorenstein of index $r$, that is, $r$ is the minimal $r \in \mathbb{N}_{>0}$ such that $r K_{X}$ is a Cartier divisor. $X$ is called Gorenstein if $r=1$.

Let $L$ be an ample line bundle (we will often use the same symbol for the associated Weil divisor) on $X$. We use the additive notation to denote the tensor 
operation in the Picard group $\operatorname{Pic}(X)$. When we consider (associated) $\mathbb{Q}$-divisors, the same additive notation will be used for the operation in the group $\operatorname{Div}(X) \otimes \mathbb{Q}$.

Recall that $L$ is $n e f$ (respectively, ample) if it has nonnegative (respectively, positive) intersection with all irreducible curves in $X$. Moreover, $L$ is said to be big if the global sections of some multiple define a birational map to a projective space. If a line bundle is nef, then being big is equivalent to having positive degree. It follows that every ample line bundle is nef and big. The pair $(X, L)$, where $L$ is an ample line bundle on $X$, is often called a polarized algebraic variety. The linear systems $\left|K_{X}+s L\right|$ are called adjoint linear systems. These systems define classical invariants which have been essential tools in the existent classification of projective varieties. In what follows we summarize what is essential to understand the results in this paper. More details can be found in [Beltrametti and Sommese 1995, 1.5.4 and 7.1.1].

Definition 4.1. Let $(X, L)$ be a polarized variety.

(1) The unnormalized spectral value of $L$ is defined as

$$
\begin{aligned}
\mu(L):=\sup \{s \in \mathbb{Q}: & h^{0}\left(N\left(K_{X}+s L\right)\right)=0 \text { for all positive integers } \\
& \text { such that } \left.N\left(K_{X}+s L\right) \text { is an integral Cartier divisor }\right\} .
\end{aligned}
$$

Note that $\mu(L)<\infty$ follows from $L$ being big.

(2) The nef value of $L$ is defined as

$$
\tau(L):=\min \left\{s \in \mathbb{R}: K_{X}+s L \text { is nef }\right\} .
$$

It was proven by Kawamata that $\tau(L) \in \mathbb{Q}$. Moreover if $r \tau=u / v$, where $u$ and $v$ are coprime, then the linear system $\left|m\left(v r K_{X}+u L\right)\right|$ is globally generated for a big enough integer $m$. The corresponding morphism

$$
f: X \rightarrow \mathbb{P}^{M}=\mathbb{P}\left(H^{0}\left(m\left(\text { vr } K_{X}+u L\right)\right)\right)
$$

has a Remmert-Stein factorization as $f=p \circ \varphi_{\tau}$, where $\varphi_{\tau}: X \rightarrow Y$ is a morphism with connected fibers onto a normal variety $Y$, called the nef value morphism. The rationality of $\mu(L)$ was only shown very recently [Birkar et al. 2010, Corollary 1.1.7] as a consequence of the existence of the minimal model program.

Observe that the invariants above can be visualized as follows - see Figure 8. Traveling from $L$ in the direction of the vector $K_{X}$ in the Neron-Severi space $\mathrm{NS}(X) \otimes \mathbb{R}$ of divisors, $L+(1 / \mu(L)) K_{X}$ is the meeting point with the cone of effective divisors $\operatorname{Eff}(X)$ and $L+(1 / \tau(L)) K_{X}$ is the meeting point with the cone of nef-divisors $\operatorname{Nef}(X)$. We now summarize some well-known results which will be used in this section.

Proposition 4.2. (1) $\tau(L)$ is the largest $s \in \mathbb{Q}$ such that $K_{X}+s L$ is nef but not ample. 


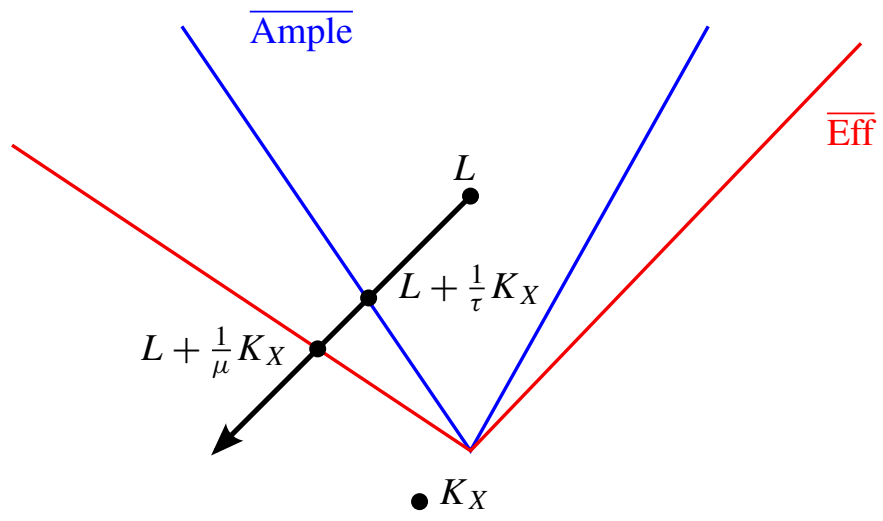

Figure 8. Illustrating $\mu(L)$ and $\tau(L)$.

(2) $\mu(L) \leqslant \tau(L)$, with equality if and only if $\varphi_{\tau}$ is not birational.

(3) Let $r(L)=u / v$ with coprime positive integers $u$, $v$. Then

$$
u \leqslant r(n+1)
$$

in particular, $\tau(L) \leqslant r(n+1)$.

(4) $\mu(L) \leqslant n+1$.

Proof. Statement (1) is proven in [Beltrametti and Sommese 1995, 1.5.5]. For (2) observe that the interior of the closure of the effective cone is the big cone, $\overline{\operatorname{Eff}(X)}^{\text {int }}=\operatorname{Big}(X)$. Recall that if a divisor is not big, then the map associated to the global sections has a lower-dimensional image. It follows that the map is birational only when $\tau$ and $v$ do not coincide. A proof can be also found in [Beltrametti and Sommese 1995, 7.1.6]. Statement (3) is part of Kawamata's rationality theorem and (4) is proven in [Beltrametti and Sommese 1995, 7.1.3].

Remark 4.3. There are at least three other notions which are related to the unnormalized spectral value. The (nonnegative) spectral value $\sigma(L):=n+1-\mu(L)$ was defined by Sommese [1986] (compare this notion with the degree of lattice polytopes [Batyrev and Nill 2007]). Fujita [1992] defined the (nonpositive) Kodaira energy $\kappa \varepsilon(L)$ as $-\mu(L)$ - see also [Batyrev and Tschinkel 1998]. Furthermore, the reciprocal $\mu(L)^{-1}$ is called the effective threshold - see, for example, [Birkar et al. 2010].

There are several classifications of polarized varieties with large nef value. For example:

Theorem 4.4 [Fujita 1987]. Let $(X, L)$ be a polarized normal Gorenstein variety with $\operatorname{dim}(X)=n$. Then: 
(1) $\tau(L) \leqslant n$ unless $(X, L)=\left(\mathbb{P}^{n}, \mathcal{O}_{\mathbb{p}}(1)\right)$.

(2) $\tau(L)<n$ unless we are in one of the following cases:

(a) $(X, L)=\left(\mathbb{P}^{n}, \mathcal{O}_{\mathbb{p} n}(1)\right)$.

(b) $X$ is a quadric hypersurface and $L=O_{X}(1)$.

(c) $(X, L)=\left(\mathbb{P}^{2}, O_{\mathbb{P}^{n}}(2)\right)$.

(d) $(X, L)=(\mathbb{P}(E), O(1))$, where $E$ is a vector bundle of rank $n$ over a nonsingular curve.

In the same paper Fujita also classifies the cases $\tau(L) \geqslant n-2$ and $\tau(L) \geqslant n-3$. We will discuss this classification in the toric setting and the induced classification of lattice polytopes with no interior lattice points in the Appendix.

Toric geometry. We refer the reader who is unfamiliar with toric geometry to [Fulton 1993]. In what follows we will assume that $X$ is a $\mathbb{Q}$-Gorenstein toric variety of Gorenstein index $r$ and dimension $n$. Let $L$ be an (equivariant) line bundle on $X$. Let $N \cong \mathbb{Z}^{n}, \Sigma \subset N \otimes \mathbb{R}$ be the defining fan and denote by $\Sigma(i)$ the set of cones of $\Sigma$ of dimension $i$. For $\tau \in \Sigma(i), V(\tau)$ will denote the associated invariant subvariety codimension $i$.

Recall that $L$ is nef (resp., ample) if and only if $L \cdot V\left(\rho_{j}\right) \geqslant 0$ (resp., >0) for all $\rho_{j} \in \Sigma(n-1)$; see [Mustaţă 2002, Theorem 3.1], for example.

There is a one-to-one correspondence between $n$-dimensional toric varieties polarized by an ample line bundle $L$ and $n$-dimensional convex lattice polytopes $P_{(X, L)} \subset M \otimes \mathbb{R}$ (up to translations by a lattice vector), where $M$ is the lattice dual to $N$. Under this correspondence $k$-dimensional invariant subvarieties of $X$ are associated with $k$-dimensional faces of $P_{(X, L)}$. More precisely, if

$$
P=\left\{x \in \mathbb{R}^{n}: A x \geqslant b\right\}
$$

for an $m \times n$ integer matrix $A$ with primitive rows and $b=\left(b_{1}, \ldots, b_{m}\right) \in \mathbb{Z}^{m}$, then $L=\sum\left(-b_{i}\right) D_{i}$, where $D_{i}=V\left(\beta_{i}\right)$, for $\beta_{i} \in \Sigma(1)$, are the invariant divisors, generating the Picard group.

More generally, a nef line bundle $\mathscr{L}$ on a toric variety $X^{\prime}$ defines a polytope $P_{\mathscr{L}} \subset \mathbb{R}^{n}$, not necessarily of maximal dimension, whose integer points correspond to characters on the torus and form a basis of $H^{0}\left(X^{\prime}, \mathscr{L}\right)$. The edges of the polytope $P_{\mathscr{L}}$ correspond to the invariant curves whose intersection with $\mathscr{L}$ is positive. In particular, the normal fan of $P_{\mathscr{L}}$ does not necessarily coincide with the fan of $X^{\prime}$. It is the fan of a toric variety $X$ obtained by possibly contracting invariant curves on $X^{\prime}$. The contracted curves correspond to the invariant curves having zero intersection with $\mathscr{L}$. Let $\pi: X^{\prime} \rightarrow X$ be the contraction morphism. There is an ample line bundle $L$ on $X$ such that $\pi^{*}(L)=\mathscr{L}$. Because the dimension of the polytope equals the dimension of the image of the map defined by the global sections one sees immediately that $P_{\mathscr{L}}$ has maximal dimension if and only if $\mathscr{L}$ is big. 
Adjoint bundles. (Compare with Section 1.) Let $(X, L)$ be the polarized variety defined by the polytope $P=\left\{x \in \mathbb{R}^{n}: A x \geqslant b\right\}$. Observe that for any $s \in \mathbb{Q}_{>0}$ the polytope $P^{(s)}:=\left\{x \in \mathbb{R}^{n}: A x \geqslant b+s \mathbb{1}\right\}$, with $\mathbb{1}=(1, \ldots, 1)^{\mathrm{T}}$, corresponds to the $\mathbb{Q}$-line bundle $s K_{X}+L$. With this interpretation it is clear that

$$
\mu(P)=\mu(L) \quad \text { and } \quad \tau(P)=\tau(L)
$$

Remark 4.5. Proposition 1.14 gives us a geometric interpretation of these invariants. Let $k \in \mathbb{Z}$ such that $k M(P)$ is a lattice polytope and let $Y$ be the associated toric variety. The polytope $P$ is a facet of $M(P)$ and thus the variety $X$ is an invariant divisor of $Y$. Moreover, the projection $M(P) \rightarrow P$ induces a rational surjective map $Y \rightarrow \mathbb{P}^{1}$ whose generic fiber (in fact all fibers but the one at $\infty$ ) are isomorphic to $X$.

Remark 4.6. From an inductive viewpoint, it would be desirable to know how "bad" the singularities of $P^{(1)}$ can get if we start out with a "nice" polytope $P$. However, this seems to be very hard. Traditionally, there is another way, the "onion skinning" of a polytope (see [Haase and Schicho 2009; Ogata 2007]) via the interior polytope $P^{[1]}:=\operatorname{conv}\left(\operatorname{int}(P) \cap \mathbb{Z}^{n}\right)$. Recall that the lattice points of $P^{(1)}$ correspond to the global sections of $K_{X}+L_{P}$. If the line bundle $K_{X}+L_{P}$ is globally generated (equivalently nef) then $P^{(1)}=P^{[1]}$, but in general they might be different. Obviously, $P^{[1]} \subseteq P^{(1)}$, with equality if and only if $P^{(1)}$ is a lattice polytope. Ogata [2007] examined the case of smooth polytopes of dimension at most three with interior lattice points. He proves the following:

- In dimension two, $P^{(1)}$ equals $P^{[1]}$, and it is even a smooth polytope [Ogata 2007, Lemma 5].

- In dimension three [Ogata 2007, Proposition 3], it is claimed that by successively forgetting facet inequalities (corresponding to blow-downs) it is possible to obtain a smooth polytope $P^{\prime} \supseteq P$ with $P^{(1)}=P^{(1)}=P^{[1]}$ and $\tau\left(P^{\prime}\right) \leqslant 1$. Moreover, while $P^{[1]}$ may not be smooth anymore, Proposition 4 of [Ogata $2007]$ says that singular points of cones over $\left(\mathbb{P}^{2}, O(2)\right)$ and $\left(\mathbb{P}^{1} \times \mathbb{P}^{1}, O(1,1)\right)$ are the only possible singularities, occurring at the toric fixpoints of $X_{P^{[1]}}$.

It would be desirable to understand what happens in higher dimensions; for instance, we expect the answer to the following question to be negative:

Let $P$ be a smooth four-dimensional polytope with interior lattice points. Is $P^{(1)}$ still a lattice polytope?

Admissible polarized toric varieties (compare with Section 2). In the language above, Proposition 2.3 states that if $(X, L)$ is a polarized $\mathbb{Q}$-Gorenstein toric variety then there is a finite sequence of maps of toric varieties

$$
X_{k} \rightarrow X_{k-1} \rightarrow \cdots \rightarrow X_{2} \rightarrow X_{1} \rightarrow X_{0}=X
$$


polarized by ample line bundles $L_{i}$. In fact, by considering the polytope $P=P_{(X, L)}$, Proposition 2.3 gives a projection $P \rightarrow Q$ from the linear space $\operatorname{Aff}\left(P^{(1 / \mu(L))}\right)$. The projection defines a map of fans $\Sigma_{Q} \rightarrow \Sigma_{P}$, and in turn a map of toric varieties $X_{1} \rightarrow X$. Notice that $\operatorname{dim}\left(X_{1}\right)=\operatorname{dim}(X)-\operatorname{dim}\left(P^{(1 / \mu(L))}\right)$. Let $L_{1}$ be the polarization defined by $Q$ on $X_{1}$. Starting again with $\left(X_{1}, L_{1}\right)$ we look at the corresponding projection $Q \rightarrow Q_{1}$ and so on. Notice that the sequence will stop when $\mu\left(X_{k-1}\right)=\mu\left(X_{k}\right)$ and $\operatorname{core}\left(Q_{k}\right)$ is a single (rational) point. We remark that the $\mathbb{Q}$-codegree has been defined for any polytope while the spectral value is defined only for $\mathbb{Q}$-Gorenstein varieties. In more generality the singularities are quite subtle and it is not at all clear how to proceed within algebraic geometry. For this purpose we will call a polarized $\mathbb{Q}$-Gorenstein toric variety admissible if in the sequence above $X_{i}$ is $\mathbb{Q}$-Gorenstein for every $0 \leqslant i \leqslant k$. Recall that the lattice points of $N \operatorname{core}\left(Q_{k}\right)$ correspond to the global sections $H^{0}\left(N\left(K_{X_{k}}+\mu\left(L_{k}\right) L_{K}\right)\right)$, for an integer $N$ such that $N\left(K_{X_{k}}+\mu\left(L_{k}\right) L_{K}\right)$ is an integral line bundle. Then Proposition 2.3 reads as follows:

Proposition 4.7. Let $(X, L)$ be an admissible polarized $\mathbb{Q}$-Gorenstein toric variety. There is a finite sequence of maps of toric varieties

$$
X_{k} \rightarrow X_{k-1} \rightarrow \cdots \rightarrow X_{2} \rightarrow X_{1} \rightarrow X_{0}=X
$$

polarized by ample line bundles $L_{i}$ such that $\mu\left(L_{i}\right) \geqslant \mu\left(L_{i-1}\right)$ for $1 \leqslant i \leqslant k$ and $H^{0}\left(N\left(K_{X_{k}}+\mu\left(L_{k}\right) L_{K}\right)\right)$ consists of a single section for an integer $N$ such that $N\left(K_{X_{k}}+\mu\left(L_{k}\right) L_{K}\right)$ is an integral line bundle.

Example 4.8. The polytope in Figure 6 defines an admissible polarized $\mathbb{Q}$-Gorenstein toric variety. Let $(X, L)$ be the associated polarized toric variety. The (unnormalized) spectral value satisfies $\mu(L)=\mu(P)=\frac{3}{4}$. The polytope has the following description:

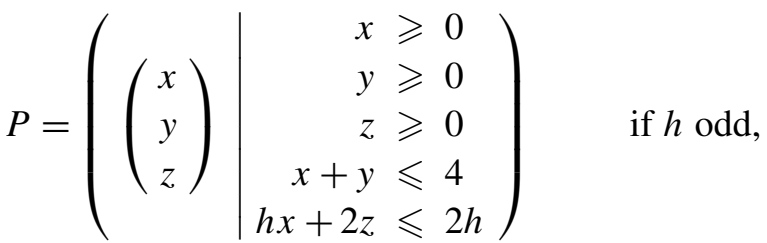

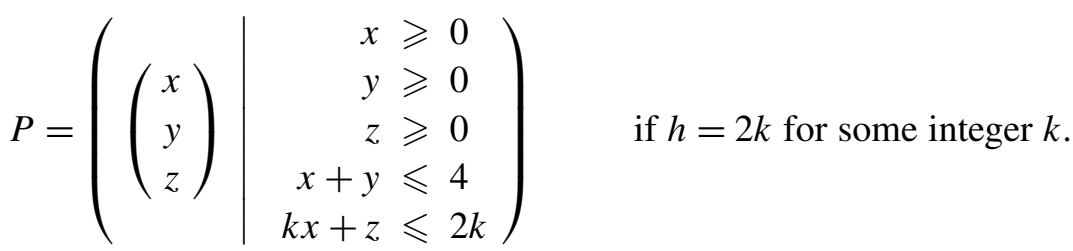

For simplicity let us assume that $h$ is odd. From the polytope one sees that $\operatorname{Pic}(X)$ is generated by $D_{1}, \ldots, D_{5}$ with the linear relations 


$$
D_{1} \sim h D_{5}+D_{4}, \quad D_{2} \sim D_{4}, \quad D_{3} \sim 2 D_{5}
$$

Moreover, $L=4 D_{4}+2 h D_{5}$ and $K_{X}=-3 D_{4}-(h+3) D_{5}$, giving $4 K_{X}+3 L=$ $(2 h-12) D_{5}$, which is effective for $h \geqslant 6$. The first projection onto $Q$ defines in this case an invariant subvariety $X_{1}$ which is isomorphic to $\mathbb{P}^{2}$ blown up at one point. Moreover $L_{1}=\left.L\right|_{X_{1}}=4 l-2 E$, where $l$ is the pull back of the hyperplane line bundle on $\mathbb{P}^{2}$ and $E$ is the exceptional divisor. The variety $X_{1}$ is smooth and therefore $\mathbb{Q}$-Gorenstein of index 1 . Starting again with $\left(X_{1}, L_{1}\right)$ we have $v\left(L_{1}\right)=1$ and $X_{2} \cong \mathbb{P}^{1}$ with $L_{2}=\mathbb{O}_{\mathbb{P}^{1}}(2)$, which give $\mu\left(L_{2}\right)=1$ and $H^{0}\left(K_{X_{2}}+L_{2}\right)=H^{0}\left(\mathcal{O}_{X_{2}}\right)$.

It would be desirable to have criteria for a toric polarized $\mathbb{Q}$-Gorenstein variety to be admissible.

The main result. (Compare with Section 3.) As explained in [Haase et al. 2009; Dickenstein et al. 2009] the toric variety $X$, defined by a Cayley polytope

$$
P=P_{0} * \cdots * P_{t},
$$

has a prescribed birational morphism to the toric projectivized bundle

$$
X=\mathbb{P}\left(H_{0} \oplus H_{1} \oplus \cdots \oplus H_{t}\right)
$$

over a toric variety $Y$. The variety $Y$ is defined by a common refinement of the inner normal fans of the polytopes $P_{i}$. Moreover, the polytopes $P_{i}$ are associated to the nef line bundles $H_{i}$ over $Y$. As a consequence of Theorem 3.4 we get the following result.

Proposition 4.9. Let $(X, L)$ be a polarized $\mathbb{Q}$-Gorenstein toric variety. Suppose $q \in \mathbb{Q}_{>0}$ such that $2 q \leqslant n$ and no multiple of $K_{X}+(n+1-q) L$ which is Cartier has nonzero global sections. Then there is a proper birational toric morphism $\pi: X^{\prime} \rightarrow X$, where $X^{\prime}$ is the projectivization of a sum of line bundles on a toric variety of dimension at most $\lfloor 2 q\rfloor$ and $\pi^{*} L$ is isomorphic to $O(1)$.

Proof. The assumption $2 q \leqslant n$ implies that $\mu(L) \geqslant(n+2) / 2$. Theorem 3.4 gives the conclusion.

Remark 4.10. It is conjectured on page 2434 that $\mu(L)>(n+1) / 2$ should suffice in Corollary 3.7. One algebro-geometric statement which hints at this possibility is a conjecture by Beltrametti and Sommese [1995, 7.1.8] stating that $\mu(L)>(n+1) / 2$ should imply $\mu(L)=\tau(L)$ when the variety is nonsingular. Moreover, it was also conjectured in [Fania and Sommese 1989] that if $\mu(L)>1$, then $\mu(L)=p / q$ for integers $0<q \leqslant p \leqslant n+1$. In particular, $\mu(L)>(n+1) / 2$ would again imply $\mu(L) \in \mathbb{Z}$.

Let $A$ be the set of lattice points of a lattice polytope $P$, and let $X_{A}$ be the (not necessarily normal) toric variety embedded in $\mathbb{P}^{|A|-1}$. Then there is an irreducible 
polynomial, called the A-discriminant, which is of degree zero if and only if the dual variety $X_{A}^{*}$ is not a hypersurface (that is, $X_{A}$ has dual defect) - see [Gelfand et al. 1994].

Proposition 4.11. Let $P$ be a lattice polytope with $\mu(P) \geqslant(3 n+4) / 4$ if $\mu(P) \notin \mathbb{N}$, or $\mu(P) \geqslant(3 n+3) / 4$ if $\mu(P) \in \mathbb{N}$. Then $X_{A}$ has dual defect.

Proof. By Theorem 3.4, $P$ is a Cayley polytope of at least $n+1-d$ lattice polytopes in $\mathbb{R}^{d}$, where the assumptions yield that $n+1-d \geqslant d+2$. Then Proposition 6.1 and Lemma 6.3 in [Dickenstein et al. 2007] imply the desired result. Note that in the notation of [Dickenstein et al. 2007] $m=n+1-d, r=d$, and $c=m-r \geqslant 2$.

For smooth polarized toric varieties it was verified that the assumption $\mu(L)>$ $(n+2) / 2$ is equivalent to the variety having dual defect [Dickenstein and Nill 2010]. Moreover, smooth dual-defective varieties are necessarily $\mathbb{Q}$-normal $(\mu(L)=\tau(L))$ by [Beltrametti et al. 1992]. By the results of [Di Rocco 2006; Dickenstein et al. 2009] this implies that the associated lattice polytope is a smooth Cayley polytope with $\mu(L)=\operatorname{cd}(P)$ factors which all share the same normal fan. On the other hand, it has recently been shown [Curran and Cattani 2007; Esterov 2010] that all lattice points in a (possibly singular) dual-defective polytope have to lie on two parallel hyperplanes. However, it is not true that all Cayley polytopes, or polytopes of lattice width 1 , are dual defective, even in the nonsingular case. Therefore, the main question is whether the following strengthening of Proposition 4.11 may be true — see [Dickenstein and Nill 2010]:

Question 4.12. Is $(X, L)$ dual defective if $\mu(L)>(n+2) / 2$ ?

\section{Appendix: Fujita's classification results}

In this section we provide a translation of the results in [Fujita 1987, Theorem 2 and $3^{\prime}$ ]. A straightforward corollary gives the classification of smooth polytopes of dimension three with no interior lattice points. One could derive a more extensive classification from the theorems just cited and from later work such as [Beltrametti and Di Termini 2003; Nakamura 1997]. This would require a more elaborate explanation, which goes beyond the scope of this paper.

Theorem A.1 [Fujita 1987]. Let $P$ be an $n$-dimensional lattice polytope such that its normal fan is Gorenstein.

(1) If $\tau(P)>n$, then $P \cong \Delta_{n}$.

(2) If $n-1<\tau(P) \leqslant n$, then $P \cong 2 \Delta_{2}$ or $P \cong P_{0} * P_{1} * \cdots * P_{n-1}$, where the $P_{i}$ are parallel intervals.

(3) If $P$ is smooth and $n-2<\tau(P) \leqslant n-1$, then $P$ is one of these polytopes: 
(a) There is a smooth $n$-dimensional polytope $P^{\prime}$ and a unimodular simplex $S \nsubseteq P$ such that

$$
P^{\prime}=P \cup S
$$

and $P \cap S$ is a common facet of $P$ and $S$.

(b) $P^{(1 /(n-1))}$ is a point.

(c) $P=2 \Delta_{3}, 3 \Delta_{3}, 2 \Delta_{4}$.

(d) There is a projection $\pi: P \rightarrow \Delta_{1} \times \Delta_{1}$

(e) There is a projection $\pi: P \rightarrow 2 \Delta_{2}$ and the polytopes $\pi^{-1}\left(m_{i}\right)$ have the same normal fan, where $m_{i}$ are the vertices of $2 \Delta_{2}$.

(f) $P \cong P_{0} * P_{1} * \cdots * P_{n-2}$, where the $P_{i}$ are smooth polygons with the same normal fan.

Note that in (3)(a) $P$ is given by a vertex truncation of $P^{\prime}$ (compare with Figure 2), corresponding to a blow-up at a smooth point. The following result is a simple corollary of the previous classification. It was also obtained in a slightly weaker form by Ogata [2007, Proposition 1], using combinatorial methods.

Corollary 4.2. Let $P$ be a smooth 3-dimensional polytope with no interior lattice points. Then $P$ is of one of the following types.

(1) $P=\Delta_{3}, 2 \Delta_{3}, 3 \Delta_{3}$.

(2) There is a projection $P \rightarrow \Delta_{2}$, where any preimage of each vertex is an interval. Equivalently, there are $a, b, c \in \mathbb{Z}$ such that

$$
P=\operatorname{conv}\left[\begin{array}{llllll}
0 & 0 & 0 & a & b & c \\
0 & 1 & 0 & 1 & 0 & 0 \\
0 & 0 & 1 & 0 & 0 & 1
\end{array}\right] .
$$

(3) There is a projection $P \rightarrow 2 \Delta_{2}$, where any preimage of each vertex is an interval. Equivalently, there are $a, b, c \in \mathbb{Z}$ such that

$$
P=\operatorname{conv}\left[\begin{array}{llllll}
0 & 0 & 0 & a & b & c \\
0 & 2 & 0 & 2 & 0 & 0 \\
0 & 0 & 2 & 0 & 0 & 2
\end{array}\right] \text {. }
$$

(4) There is a projection $P \rightarrow \Delta_{1} \times \Delta_{1}$. Equivalently, there are $a, b, c \in \mathbb{Z}$ such that

$$
P=\operatorname{conv}\left[\begin{array}{cccccccc}
0 & 0 & 1 & 1 & 0 & 1 & 1 & 0 \\
0 & 1 & 0 & 1 & 1 & 1 & 0 & 0 \\
0 & 0 & 0 & 0 & a & b & c & a+b-c
\end{array}\right] .
$$

(5) $P=P_{0} * P_{1}$, where $P_{0}$ and $P_{1}$ are smooth polygons with the same normal fan.

(6) There is a smooth 3-dimensional polytope $P^{\prime}$ with no interior lattice points and a unimodular simplex $S \nsubseteq P$ such that

$$
P^{\prime}=P \cup S
$$

and $P \cap S$ is a common facet of $P$ and $S$. 


\section{Acknowledgements}

This work was carried out when several of the authors met at FU Berlin, KTH Stockholm and the Institut Mittag-Leffler. The authors would like to thank these institutions and the Göran Gustafsson foundation for hospitality and financial support.

We thank Sam Payne for pointing out the reference [Fujita 1987], Michael Burr for exhibiting the relation to the straight skeleton and Alicia Dickenstein for the proof of Proposition 4.11. Finally, we would like to thank the anonymous referees for several suggestions that led to improvements and clarifications.

\section{References}

[Aichholzer et al. 1995] O. Aichholzer, F. Aurenhammer, D. Alberts, and B. Gärtner, "A novel type of skeleton for polygons", J. UCS 1:12 (1995), 752-761. MR 97c:52028 Zbl 0943.68171

[Averkov et al. 2011] G. Averkov, C. Wagner, and R. Weismantel, "Maximal lattice-free polyhedra: finiteness and an explicit description in dimension three", Math. Oper. Res. 36:4 (2011), 721-742. MR 2012j:90103 Zbl 1246.90107

[Barvinok 2002] A. Barvinok, A course in convexity, Graduate Studies in Mathematics 54, Amer. Math. Soc., Providence, RI, 2002. MR 2003j:52001 Zbl 1014.52001

[Batyrev and Nill 2007] V. V. Batyrev and B. Nill, "Multiples of lattice polytopes without interior lattice points", Mosc. Math. J. 7:2 (2007), 195-207. MR 2008g:52018 Zbl 1134.52020

[Batyrev and Nill 2008] V. V. Batyrev and B. Nill, "Combinatorial aspects of mirror symmetry", pp. 35-66 in Integer points in polyhedra: geometry, number theory, representation theory, algebra, optimization, statistics (Snowbird, UT, 2006), edited by M. Beck et al., Contemp. Math. 452, Amer. Math. Soc., Providence, RI, 2008. MR 2009m:14059 Zbl 1161.14037

[Batyrev and Tschinkel 1998] V. V. Batyrev and Y. Tschinkel, "Tamagawa numbers of polarized algebraic varieties", pp. 299-340 in Nombre et répartition de points de hauteur bornée (Paris, 1996), edited by E. Peyre, Astérisque 251, Société Mathématique de France, Paris, 1998. MR 2000d:11090 Zbl 0926.11045

[Beck and Robins 2007] M. Beck and S. Robins, Computing the continuous discretely: integer-point enumeration in polyhedra, Springer, New York, 2007. MR 2007h:11119 Zbl 1114.52013

[Beltrametti and Di Termini 2003] M. C. Beltrametti and S. Di Termini, "Higher dimensional polarized varieties with non-integral nef value", Adv. Geom. 3:3 (2003), 287-299. MR 2004j:14006 Zbl 1053.14061

[Beltrametti and Sommese 1994] M. C. Beltrametti and A. J. Sommese, "Some effects of the spectral values on reductions”, pp. 31-48 in Classification of algebraic varieties (L'Aquila, 1992), edited by C. Ciliberto et al., Contemp. Math. 162, Amer. Math. Soc., Providence, RI, 1994. MR 95d:14006 Zbl 0829.14003

[Beltrametti and Sommese 1995] M. C. Beltrametti and A. J. Sommese, The adjunction theory of complex projective varieties, de Gruyter Expositions in Mathematics 16, de Gruyter, Berlin, 1995. MR 96f:14004 Zbl 0845.14003

[Beltrametti et al. 1992] M. C. Beltrametti, M. L. Fania, and A. J. Sommese, "On the discriminant variety of a projective manifold”, Forum Math. 4:6 (1992), 529-547. MR 93k:14049 Zbl 0780.14023 
[Birkar et al. 2010] C. Birkar, P. Cascini, C. D. Hacon, and J. McKernan, "Existence of minimal models for varieties of log general type”, J. Amer. Math. Soc. 23:2 (2010), 405-468. MR 2011f:14023 Zbl 1210.14019

[Curran and Cattani 2007] R. Curran and E. Cattani, "Restriction of $A$-discriminants and dual defect toric varieties”, J. Symbolic Comput. 42:1-2 (2007), 115-135. MR 2007j:14076 Zbl 1167.14326

[Debarre 2003] O. Debarre, "Fano varieties", pp. 93-132 in Higher dimensional varieties and rational points (Budapest, 2001), edited by K. J. Böröczky et al., Bolyai Soc. Math. Stud. 12, Springer, Berlin, 2003. MR 2004g:14045 Zbl 0939.14022

[Di Rocco 2006] S. Di Rocco, "Projective duality of toric manifolds and defect polytopes", Proc. London Math. Soc. (3) 93:1 (2006), 85-104. MR 2007d:14093 Zbl 1098.14039

[Dickenstein and Nill 2010] A. Dickenstein and B. Nill, "A simple combinatorial criterion for projective toric manifolds with dual defect", Math. Res. Lett. 17:3 (2010), 435-448. MR 2011f:52019 Zbl 1243.52010

[Dickenstein et al. 2007] A. Dickenstein, E. M. Feichtner, and B. Sturmfels, "Tropical discriminants", J. Amer. Math. Soc. 20:4 (2007), 1111-1133. MR 2008j:14095 Zbl 1166.14033

[Dickenstein et al. 2009] A. Dickenstein, S. Di Rocco, and R. Piene, "Classifying smooth lattice polytopes via toric fibrations", Adv. Math. 222:1 (2009), 240-254. MR 2010d:52027 Zbl 1193.14065

[Eppstein and Erickson 1999] D. Eppstein and J. Erickson, "Raising roofs, crashing cycles, and playing pool: applications of a data structure for finding pairwise interactions", Discrete Comput. Geom. 22:4 (1999), 569-592. MR 2000i:68185 Zbl 0946.68147

[Esterov 2010] A. Esterov, "Newton polyhedra of discriminants of projections", Discrete Comput. Geom. 44:1 (2010), 96-148. MR 2011j:52029 Zbl 05723617

[Fania and Sommese 1989] M. L. Fania and A. J. Sommese, "On the projective classification of smooth $n$-folds with $n$ even”, Ark. Mat. 27:2 (1989), 245-256. MR 90j:14051 Zbl 0703.14005

[Fujita 1987] T. Fujita, "On polarized manifolds whose adjoint bundles are not semipositive”, pp. 167-178 in Algebraic geometry (Sendai, 1985), edited by T. Oda, Adv. Stud. Pure Math. 10, Kinokuniya/North-Holland, Tokyo/Amsterdam, 1987. MR 89d:14006 Zbl 0659.14002

[Fujita 1992] T. Fujita, "On Kodaira energy and adjoint reduction of polarized manifolds", Мanuscripta Math. 76:1 (1992), 59-84. MR 93i:14032 Zbl 0766.14027

[Fujita 1996] T. Fujita, "On Kodaira energy of polarized log varieties", J. Math. Soc. Japan 48:1 (1996), 1-12. MR 97b:14018 Zbl 0914.14001

[Fujita 1997] T. Fujita, “On Kodaira energy and adjoint reduction of polarized threefolds", Мапuscripta Math. 94:2 (1997), 211-229. MR 99a:14007 Zbl 0969.14001

[Fulton 1993] W. Fulton, Introduction to toric varieties: the William H. Roever lectures in geometry, Annals of Mathematics Studies 131, Princeton University Press, Princeton, NJ, 1993. MR 94g:14028 Zbl 0813.14039

[Gelfand et al. 1994] I. M. Gelfand, M. M. Kapranov, and A. V. Zelevinsky, Discriminants, resultants, and multidimensional determinants, Birkhäuser, Boston, 1994. MR 95e:14045 Zbl 0827.14036

[Haase and Schicho 2009] C. Haase and J. Schicho, "Lattice polygons and the number $2 i+7$ ", Amer. Math. Monthly 116:2 (2009), 151-165. MR 2010b:52017 Zbl 1193.14066

[Haase et al. 2009] C. Haase, B. Nill, and S. Payne, "Cayley decompositions of lattice polytopes and upper bounds for $h^{*}$-polynomials", J. Reine Angew. Math. 637 (2009), 207-216. MR 2011d:52022 Zbl 1185.52012

[Joswig et al. 2009] M. Joswig, B. Müller, and A. Paffenholz, "polymake and lattice polytopes", pp. 491-502 in 21 st International Conference on Formal Power Series and Algebraic Combinatorics 
(FPSAC 2009) (Hagenberg, 2009), edited by C. Krattenthaler et al., Assoc. Discrete Math. Theor. Comput. Sci., Nancy, 2009. MR 2011g:52028 arXiv 0902.2919

[Morrison and Stevens 1984] D. R. Morrison and G. Stevens, "Terminal quotient singularities in dimensions three and four", Proc. Amer. Math. Soc. 90:1 (1984), 15-20. MR 85a:14004 Zbl 0536.14003

[Mustaţă 2002] M. Mustaţă, "Vanishing theorems on toric varieties", Tohoku Math. J. (2) 54:3 (2002), 451-470. MR 2003e:14013 Zbl 1092.14064

[Nakamura 1997] S. Nakamura, "On the classification of the third reduction with a spectral value condition”, J. Math. Soc. Japan 49:4 (1997), 633-646. MR 98g:14016 Zbl 0932.14023

[Nill 2005] B. Nill, “Gorenstein toric Fano varieties”, Manuscripta Math. 116:2 (2005), 183-210. MR 2005k:14110 Zbl 1067.14052

[Nill 2008] B. Nill, "Lattice polytopes having $h^{*}$-polynomials with given degree and linear coefficient”, European J. Combin. 29:7 (2008), 1596-1602. MR 2009f:52029 Zbl 1149.52013

[Nill and Ziegler 2011] B. Nill and G. M. Ziegler, "Projecting lattice polytopes without interior lattice points”, Math. Oper. Res. 36:3 (2011), 462-467. MR 2012k:52034 Zbl 1243.52012

[Ogata 2007] S. Ogata, "Projective normality of nonsingular toric varieties of dimension three", preprint, 2007. arXiv 0712.0444

[Sommese 1986] A. J. Sommese, "On the adjunction theoretic structure of projective varieties", pp. 175-213 in Complex analysis and algebraic geometry (Göttingen, 1985), edited by H. Grauert, Lecture Notes in Math. 1194, Springer, Berlin, 1986. MR 87m:14049 Zbl 0601.14029

[Stanley 1993] R. P. Stanley, "A monotonicity property of $h$-vectors and $h^{*}$-vectors", European J. Combin. 14:3 (1993), 251-258. MR 94f:52016 Zbl 0799.52008

[Ziegler 1995] G. M. Ziegler, Lectures on polytopes, Graduate Texts in Mathematics 152, Springer, New York, 1995. MR 96a:52011 Zbl 0823.52002

Communicated by Bernd Sturmfels

Received 2012-06-27 Revised 2012-11-02 Accepted 2013-03-16

dirocco@math.kth.se Department of Mathematics, KTH, SE-10044 Stockholm, Sweden

haase@math.uni-frankfurt.de Institut für Mathematik, Goethe-Universität Frankfurt, Robert-Mayer-Str. 10, D-60325 Frankfurt, Germany

nill@math.su.se Department of Mathematics, Stockholm University, SE 10691 Stockholm, Sweden

paffenholz@mathematik.tu-darmstadt.de

Fachbereich Mathematik, Technische Universität Darmstadt, Dolivostr. 15, D-64293 Darmstadt, Germany 


\section{Algebra \& Number Theory}

msp.org/ant

\section{EDITORS}

MANAGING EDITOR

Bjorn Poonen

Massachusetts Institute of Technology

Cambridge, USA

\author{
EDITORIAL BOARD CHAIR \\ David Eisenbud \\ University of California \\ Berkeley, USA
}

\section{BOARD OF EDITORS}

Georgia Benkart

Dave Benson

Richard E. Borcherds

John H. Coates

J-L. Colliot-Thélène

Brian D. Conrad

Hélène Esnault

Hubert Flenner

Edward Frenkel

Andrew Granville

Joseph Gubeladze

Roger Heath-Brown

Ehud Hrushovski

Craig Huneke

Mikhail Kapranov

Yujiro Kawamata

János Kollár

Yuri Manin

Barry Mazur

Philippe Michel
University of Wisconsin, Madison, USA

University of Aberdeen, Scotland

University of California, Berkeley, USA

University of Cambridge, UK

CNRS, Université Paris-Sud, France

University of Michigan, USA

Freie Universität Berlin, Germany

Ruhr-Universität, Germany

University of California, Berkeley, USA

Université de Montréal, Canada

San Francisco State University, USA

Oxford University, UK

Hebrew University, Israel

University of Virginia, USA

Yale University, USA

University of Tokyo, Japan

Princeton University, USA

Northwestern University, USA

Harvard University, USA

École Polytechnique Fédérale de Lausanne
Susan Montgomery

Shigefumi Mori

Raman Parimala

Jonathan Pila

Victor Reiner

Karl Rubin

Peter Sarnak

Joseph H. Silverman

Michael Singer

Vasudevan Srinivas

J. Toby Stafford

Bernd Sturmfels

Richard Taylor

Ravi Vakil

Michel van den Bergh

Marie-France Vignéras

Kei-Ichi Watanabe

Efim Zelmanov

Shou-Wu Zhang
University of Southern California, USA

RIMS, Kyoto University, Japan

Emory University, USA

University of Oxford, UK

University of Minnesota, USA

University of California, Irvine, USA

Princeton University, USA

Brown University, USA

North Carolina State University, USA

Tata Inst. of Fund. Research, India

University of Michigan, USA

University of California, Berkeley, USA

Harvard University, USA

Stanford University, USA

Hasselt University, Belgium

Université Paris VII, France

Nihon University, Japan

University of California, San Diego, USA

Princeton University, USA

PRODUCTION

production@msp.org

Silvio Levy, Scientific Editor

See inside back cover or msp.org/ant for submission instructions.

The subscription price for 2013 is US $\$ 200 /$ year for the electronic version, and $\$ 350 /$ year $(+\$ 40$, if shipping outside the US) for print and electronic. Subscriptions, requests for back issues and changes of subscribers address should be sent to MSP.

Algebra \& Number Theory (ISSN 1944-7833 electronic, 1937-0652 printed) at Mathematical Sciences Publishers, 798 Evans Hall \#3840, c/o University of California, Berkeley, CA 94720-3840 is published continuously online. Periodical rate postage paid at Berkeley, CA 94704, and additional mailing offices.

ANT peer review and production are managed by EditFLOW ${ }^{\circledR}$ from Mathematical Sciences Publishers.

\section{PUBLISHED BY}

- mathematical sciences publishers

nonprofit scientific publishing

http://msp.org/

(C) 2013 Mathematical Sciences Publishers 


\section{Algebra \& Number Theory}

Volume $7 \quad$ No. $10 \quad 2013$

On Kato's local $\epsilon$-isomorphism conjecture for rank-one Iwasawa modules

OTMAR VENJAKOB

Polyhedral adjunction theory

SANDRA Di Rocco, Christian HaAse, Benjamin Nill and ANDREas

PAFFENHOLZ

Genericity and contragredience in the local Langlands correspondence

TASHO KALETHA

Homogeneous projective bundles over abelian varieties

MICHEL BRION

On the second Tate-Shafarevich group of a 1-motive

PETER JOSSEN

Triangulable $\mathcal{O}_{F}$-analytic $\left(\varphi_{q}, \Gamma\right)$-modules of rank 2

LIONEL FoURQUAUX and BINGYONG XIE 